\title{
Basic Local Alignment Search Tool
}

Jump to Page Content

\section{Tax BLAST Report}

\section{Index}

- Lineage Report

- Organism Report

- Taxonomy Report

- $\underline{\text { Help }}$

Lineage Report

root

Fungi/Metazoa group [eukaryotes]

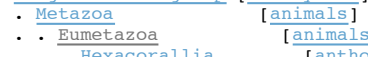

$\therefore$. Hexacorallia $\quad$ [anthozoans]

. . . Acropora millepora ---

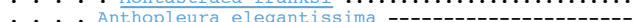

Nematostella vectensisin.

. . Microcosmus squamiger --

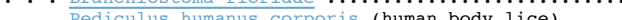

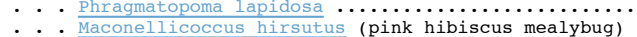

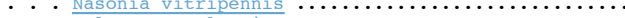

$\therefore$ Solea senegalensis

$\therefore$ Ixodes scapularis (shoulder tick)

$\therefore$ Dermacentor variabilis

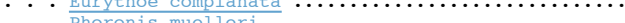

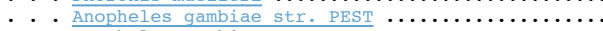

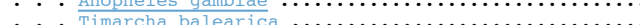

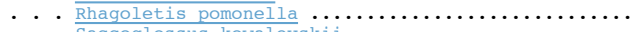

Macaca mulatta (rhesus macaque)

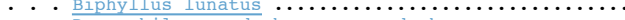

$\therefore$ Drosophila pseudoobscura pseudoobscura $\cdots \cdots \ldots \ldots$

.. Sarcophaga crassipalpis

. Drosophila mojavensi

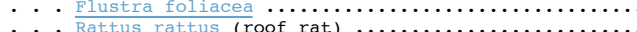

. . Mustela putorius furo (ferret)

$\therefore$. Mus musculus (mouse) $\ldots . . . .$.

... Homo sapiens (man)

- Trichoplax adhaeren

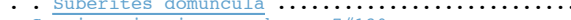

- Coprinopsis cinerea okayama7\#130

- Laccaria bicolor $\mathrm{S} 238 \mathrm{~N}-\mathrm{H} 82$

synthetic construct
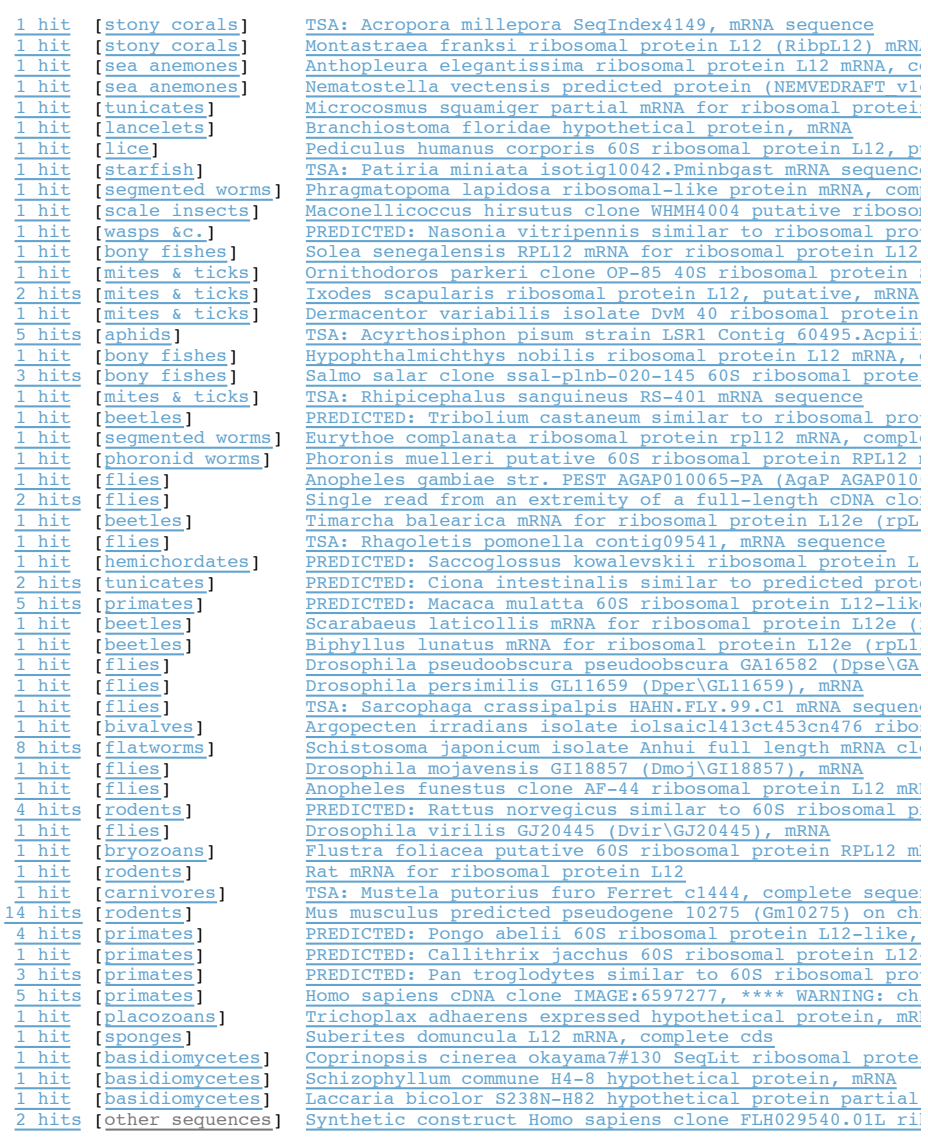

\section{Organism Report}

Acropora millepora [stony corals] taxid 45264

gb|EZ024706.1| TSA: Acropora millepora SeqIndex4149, mRNA ...

Anthopleura elegantissima [sea anemones] taxid 6110

gb|DQ314616.1| Anthopleura elegantissima ribosomal protein...

Microcosmus squamiger [tunicates] taxid 439822

emb|FN984766.1| Microcosmus squamiger partial mRNA for rib...

Branchiostoma floridae [lancelets] taxid 7739

ref|XM 002591796.1| Branchiostoma floridae hypothetical pr...

Pediculus humanus corporis (human body lice) [lice] taxid 121224

ref|XM 002425415.1| Pediculus humanus corporis 60 S ribosom..

Patiria miniata [starfish] taxid 46514

gb|HP092325.1| TSA: Patiria miniata isotig10042.Pminbgast...

Nematostella vectensis [sea anemones] taxid 45351

ref|XM_001641997.1| Nematostella vectensis predicted prote...

Phragmatopoma lapidosa [segmented worms] taxid 341668

gb|GQ455407.1| Phragmatopoma lapidosa ribosomal-like prote...

Maconellicoccus hirsutus (pink hibiscus mealybug, ...) [scale insects] taxid 177089 
Nasonia vitripennis [wasps \&C.] taxid 7425

ref|XM 001605592.1| PREDICTED: Nasonia vitripennis similar...

Solea senegalensis [bony fishes] taxid 28829

dbj|AB374947.1| Solea senegalensis RPL12 mRNA for ribosoma...

Trichoplax adhaerens [placozoans] taxid 10228

ref|XM 002108586.1| Trichoplax adhaerens expressed hypothe...

Ornithodoros parkeri [mites \& ticks] taxid 140564

gb|EF633964.1| Ornithodoros parkeri clone OP-85 40 S riboso...

Ixodes scapularis (shoulder tick, ...) [mites \& ticks] taxid 6945

gb $10066203.1 \mid$ Ixodes

Dermacentor variabilis [mites \& ticks] taxid 34621

gb|EU551645.1| Dermacentor variabilis isolate DvM 40 ribos...

0.0
0.0

Coprinopsis cinerea okayama7\#130 [basidiomycetes] taxid 24017 ref|XM 001830397.2| Coprinopsis cinerea okayama7\#130 SeqLi...

Acyrthosiphon pisum [aphids] taxid 7029

gb HP395106.1 TSA: Acyrthosiphon pisum strain LSR1 Contig... gb HP301142.1 TSA: Acyrthosiphon pisum strain LSR1 Contig... abj AK340119.1 Acyrthosiphon pisum ACYPI000059 mRNA, clon... ef NM 001126171.2 Acyrthosiphon pisum ribosomal protein ... 16025.1 Acyrthosiphon pisum putative ribosomal prot...

Hypophthalmichthys nobilis (noble carp, ...) [bony fishes] taxid 7965 gb|HM146124.1| Hypophthalmichthys nobilis ribosomal protei...

Salmo salar [bony fishes] taxid 8030

gb|BT048569.2 Salmo salar clone ssal-plnb-020-145 60S rib...

Salmo salar clone ssal-rgb2-526-129 60 S rib... Salmo salar clone HM6 0646 ribosomal protei...

Rhipicephalus sanquineus [mites \& ticks] taxid 34632

gb|EZ406119.1| TSA: Rhipicephalus sanguineus RS-401 mRNA s...

Tribolium castaneum (rust-red flour beetle) [beetles] taxid 7070 ref|XM 970437.2 PREDICTED: Tribolium castaneum similar to...

Eurythoe complanata [segmented worms] taxid 167815

gb|EU125012.1| Eurythoe complanata ribosomal protein rpl12...

Schizophyllum commune H4-8 [basidiomycetes] taxid 578458 ref XM 003029870.1 Schizophyllum commune H4-8 hypothetica...

Phoronis muelleri [phoronid worms] taxid 478209

gb|EU558362.1| Phoronis muelleri putative 60S ribosomal pr...

Anopheles gambiae str. PEST [flies] taxid 180454

ref|XM 319222.4| Anopheles gambiae str. PEST AGAP010065-PA...

Anopheles gambiae [flies] taxid 7165 \begin{tabular}{l|l|l} 
emb & BX063995.1 Single read from an extremity of a full-le... \\
emb $B \times 009066.1$ & Single read from an extremity of a full-le...
\end{tabular}

Suberites domuncula [sponges] taxid 55567

gb AY857425.1 Suberites domuncula L12 mRNA, complete cds

Timarcha balearica [beetles] taxid 79517

emb|AM049032.1| Timarcha balearica mRNA for ribosomal prot...

Rhagoletis pomonella [flies] taxid 28610

gb EZ125760.1 TSA: Rhagoletis pomonella contig09541, mRNA...

Saccoglossus kowalevskii [hemichordates] taxid 10224

ef XM 002741431.1 PREDICTED: Saccoglossus kowalevskii ri...

Ciona intestinalis [tunicates] taxid 7719

ef XM 002131525.1 PREDICTED: Ciona intestinalis similar ...

dbj AK113209.1 Ciona intestinalis CDNA, clone:ciad015019,...

Macaca mulatta (rhesus macaque, ...) [primates] taxid 9544 PREDICTED: Macaca mulatta $60 \mathrm{~S}$ ribosomal p...

ref NM 001193560.1 Macaca mulatta ribosomal protein L12 (... ref XM 001114984.2 PREDICTED: Macaca mulatta 60S ribosoma... ref XM 002801376.1 PREDICTED: Macaca mulatta 60S ribosoma..

Laccaria bicolor S238N-H82 [basidiomycetes] taxid 486041 ref|XM 001880189.1| Laccaria bicolor S238N-H82 hypothetica...

Scarabaeus laticollis [beetles] taxid 29245

emb AM049030.1| Scarabaeus laticollis mRNA for ribosomal p...

Biphyllus lunatus [beetles] taxid 197003

emb|AM049028.1| Biphyllus lunatus mRNA for ribosomal prote...

Drosophila pseudoobscura pseudoobscura [flies] taxid 46245 ref|XM 001360854.2| Drosophila pseudoobscura pseudoobscura..

Drosophila persimilis [flies] taxid 7234

ref|XM $002016543.1 \mid$ Drosophila persimilis GL11659 (Dper\GL...

Sarcophaga crassipalpis [flies] taxid 59312

gb|EZ597013.1| TSA: Sarcophaga crassipalpis HAHN.FLY.99.C1...

Argopecten irradians [bivalves] taxid 31199

gb|AF526229.1| Argopecten irradians isolate iolsaicl413ct4...

Schistosoma japonicum [flatworms] taxid 6182

mb FN315220.1 Schistosoma japonicum isolate Anhui full 1..

emb FN315219.1 Schistosoma japonicum isolate Anhui full $1 .$. 
Montastraea franksi [stony corals] taxid 48499

gb|FJ790233.1| Montastraea franksi ribosomal protein L12 (...

Drosophila mojavensis [flies] taxid 7230

ref|XM 002005813.1| Drosophila mojavensis GI18857 (Dmoj\GI...

Anopheles funestus [flies] taxid 62324

gb|D0910352.1| Anopheles funestus clone AF-44 ribosomal pr...

Rattus norvegicus (brown rat, ...) [rodents] taxid 10116

PREDICTED: Rattus norvegicus similar t... PREDICTED: Rattus norvegicus similar t...

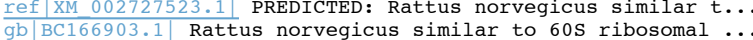

ref NM 001109198.1| Rattus norvegicus similar to 60 s ribos...

Drosophila virilis [flies] taxid 7244

ref|XM 002049947.1| Drosophila virilis GJ20445 (Dvir\GJ204...

Flustra foliacea [bryozoans] taxid 478208

gb|EU139209.1| Flustra foliacea putative $60 \mathrm{~s}$ ribosomal pro...

Rattus rattus (roof rat, ...) [rodents] taxid 10117

emb|x53504.1| Rat mRNA for ribosomal protein L12

Mustela putorius furo (ferret, ...) [carnivores] taxid 9669 gb|EZ457883.1| TSA: Mustela putorius furo Ferret_c1444, co...

Mus musculus (mouse) [rodents] taxid 10090

ref|NG 0047596 Mus musculus predicted pseudogene $10275 \quad \ldots$ ref NG 007061.2 Mus musculus predicted gene 9396 (Gm9396) ... ref NM 009076.3 Mus musculus ribosomal protein L12 (Rpl12...

Mus musculus chromosome 3, clone RP24-383A..

gb AC164078,4 Mus musculus ribosomal protein L12, mRNA (c...

gb AC166262.3 Mus musculus BAC RP23-256D10 (Roswell Park...

Mus musculus BAC RP23-256D10 (Roswell Park...

Mus musculus CRL-1722 L5178Y-R CDNA, RTIEN...

Mus musculus lung RCB-0558 LLC CDNA, RIKEN...

Mus musculus bone marrow macrophage CDNA, ...

Mus musculus bone marrow macrophage CDNA, ...

Mus musculus ribosomal protein L12, mRNA (c..

$\frac{\mathrm{gb}}{\mathrm{gb} 118620.10 \mid}$ Mus musculus chromosome 3, clone RP24-571H...

Pongo abelii (Orang-utan, ...) [primates] taxid 9601

ref|XM 002821249.1 PREDICTED: Pongo abelii 60s ribosomal ...

ef XM 002821247.1 PREDICTED: Pongo abelii 60S ribosomal ...

ref XM 002820230.1 PREDICTED: Pongo abelii 60s ribosomal ...

Callithrix jacchus (common marmoset, ...) [primates] taxid 9483 ref|XM 002754372.1 PREDICTED: Callithrix jacchus 60S ribo..

Pan troglodytes [primates] taxid 9598

ref XM 528436.2 PREDICTED: Pan troglodytes similar to 60s... ef XM 001137362.1 PREDICTED: Pan troglodytes similar to $\cdots$ ref XM 516562.2 PREDICTED: Pan troglodytes similar to rib...

Homo sapiens (man) [primates] taxid 9606

b BC085006.1 Homo sapiens CDNA clone IMAGE: 6597277, $* * * * \ldots$

b BCO71921 Homo sapiens CDNA Clone IMAGE:6423605, $* * * * \ldots$

b BC001802.1 Homo sapiens CDNA clone IMAGE:3355546, ****...

\begin{tabular}{l|l|l|l} 
emb & CR625201.1 full-length CDNA clone CS0DC011YI15 of Neu... \\
\hline emb & CR622190.1 full-length CDNA clone CS0DL009YN18 of B c..
\end{tabular}

synthetic construct [other sequences] taxid 32630

b AY891709.1 Synthetic construct Homo sapiens clone FLHO...

gb AY889175.1 Synthetic construct Homo sapiens clone FLH0..

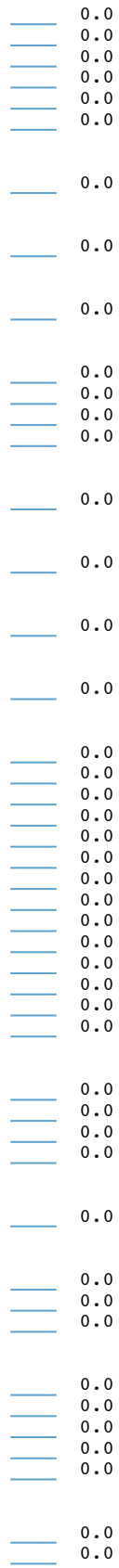

\section{Taxonomy Report}

root $\ldots \ldots \ldots \ldots \ldots \ldots$

Fungi/Metazoa

. Eumetazoa $\ldots$ Hexacorali

Scleractin

Acropora miliepor

Actiniaria

.

. Anthopleura elegantissin.

Bilateria...

Coelomata .....

Deuterostomia

Ascidiacea

Microcosmus squamige

- Ciona intestinalis

Euteleostomi

clupeocephaia

. Solea senegalensi

- Salmo salar

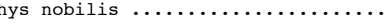

Euarchontoglires

Simiiformes

. . . . . . . . Macaca mu

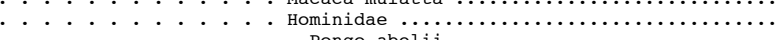

100 hits

98 hits

95 hits

4 hit

2 hits

1 hits

1 hits

2 hit

1 hits

1 hits

89 hits

81 hits

49 hits
47 hits

3 hits

1 hits

$\begin{array}{ll}2 & \text { hits } \\ 1 & \text { hits }\end{array}$

43 hits

5 hits

4 hits
1 hits

1 hits
3 hits

1 hits

38 hits

37 hits

18 hits
17 hits

5 hits

12 hits

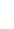

0

.0

0

0

0

0.0

0.0
0.0

.0

0

0

.0

.0

.0
要 0.0 0.0

54 orgs
53 orgs [cellular organisms; Eukaryota]
50 orgs

50 orgs
48 orgs

48 orgs [Cnidaria; Anthozoa]
4 orgs

1 orgs [Astrocoeniina; Acroporidae; Acropora]

1 orgs [Faviina; Faviidae; Montastraea]

2 orgs [Nynantheae; Actiniidae; Anthopleura]

1 orgs [Edwardsiidae; Nematostella]

44 orgs
43 orgs

43 orgs
17 orgs

17 orgs

2 orgs [Tunicata]

1 orgs [Stolidobranchia; Pyuridae; Microcosmus]

1 orgs [Enterogona; Phlebobranchia; Cionidae; Ciona]

$\begin{aligned} 1 & \text { orgs [Enterogona; Phlebobranchio; Cionidae; Ciona] } \\ 1 & \text { orgs [Cephalochordata; Branchiostomidae; Branchiostoma] } \\ 12 & \text { orgs [Craniata; Vertebrata; Gnathostomata; Teleostomi] }\end{aligned}$

3 orgs [Actinopterygi; Actinopteri; Neopterygii; Teleostei; E

2 orgs
1 orgs [Neognathi; Neoteleostei; Eurypterygii; Ctenosquamata;
1 orgs [Protacanthopterygii; Salmoniformes; Salmonoidei; Salmo:

1 orgs [Protacanthopterygii; Salmoniformes; Salmonoidei; Salmo:

orgs [Otocephala; Ostariophysi; Otophysi; Cypriniphysi; Cypr
orgs [Sarcopterygii; Tetrapoda; Amniota; Mammalia; Theria]

9 orgs
8 orgs

8 orgs [Primates; Haplorrhini]

1 orgs [Cercopithecoidea; Cercopithecidae; Cercopithecinae; Mar

3 orgs [Hominoidea]

3 orgs [Hominoidea] 


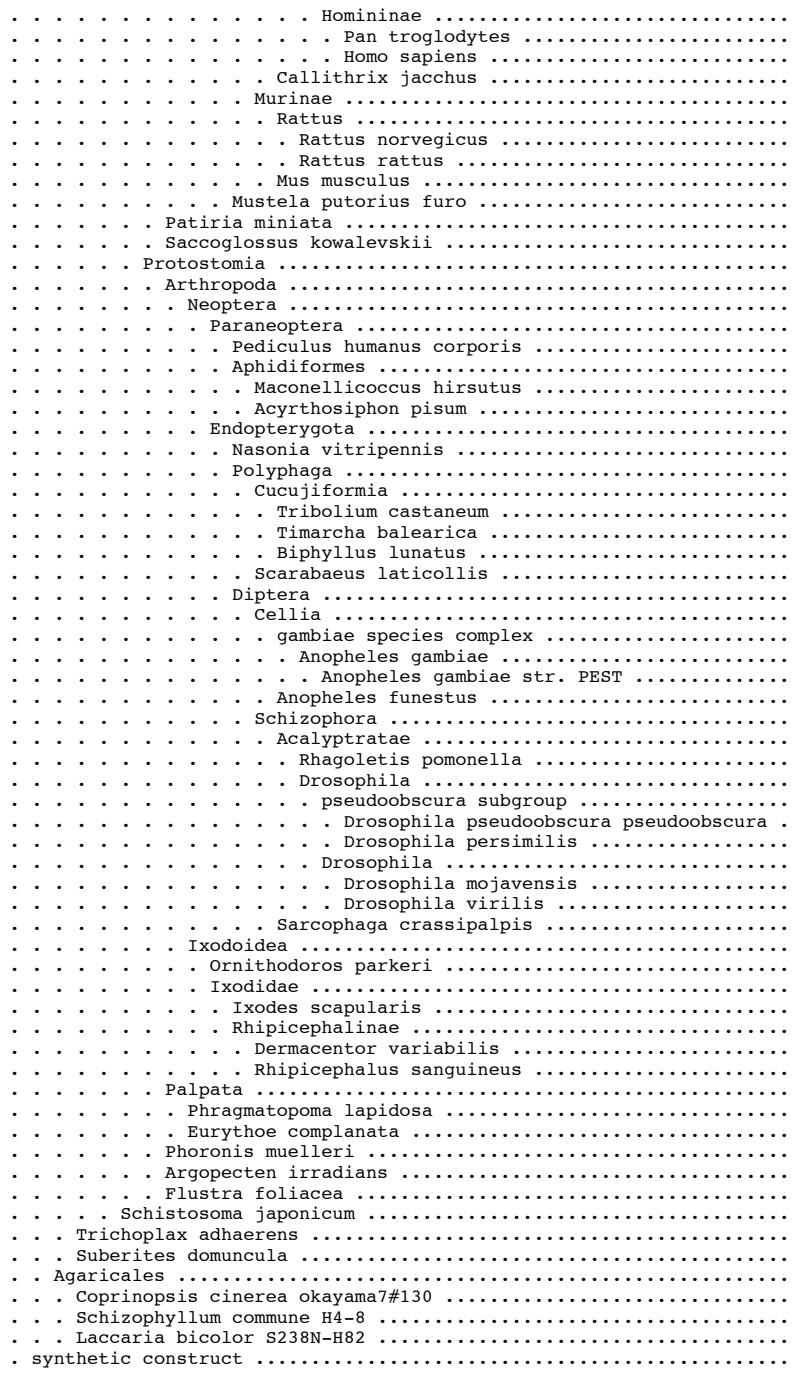

8 hits 2 orgs

3 hits 1 orgs [Pan]

1 hits 1 orgs [Homo]

19 hits 3 orgs [Glires; Rodentia; Sciurognathi; Muroidea; Muridae

5 hits 2 orgs

4 hits 1 orgs

1 hits 1 orgs

14 hits 1 orgs [Mus; Mus]

1 hits 1 orgs [Echinodermata; Eleutherozoa; Asterozoa; Asteroidea; Va

1 hits 1 orgs [Hemichordata; Enteropneusta; Harrimanidae; Saccogloss

32 hits 26 orgs

27 hits 21 orgs [Panarthropoda]

$\begin{aligned} 22 & \text { hits } \\ 7 & \text { hits }\end{aligned}$

1 hit

1 hits 1 orgs [Phthiraptera; Anoplura; Pediculidae; Pediculus; Pedicu
6 hits 2 orgs [Hemitera; Sternornyncha]
1 hits 1 orgs [Cocoidea; Pseudococcidae; Maconellicoccus]

1 orgs [Aphidomorpha; Aphidoidea; Aphididae; Aphidinae; Macros

15 hits

4 hits

3 hits

3 hits

1 hits

$\begin{aligned} 1 & \text { hits } \\ 1 & \text { hits } \\ 10 & \text { hits }\end{aligned}$

10 hits

4 hits

3 hits

3 hits
1 hits

1 hits

6 hits

5 hits
1 hits

4 hits

2 hits

1 hits

1 hits
2 hits

1 hits

1 hits

5 hits

1 hits

4 hits

2 hits

2 hits
1 hits

1 hits

$\begin{array}{ll}2 & \text { hits } \\ 1 & \text { hits }\end{array}$

1 hits

1 hits

1 hits

$\begin{array}{ll}1 & \text { hits } \\ 8 & \text { hits }\end{array}$

1 hits

1 hits

3 hits
1 hits
1

1 hits

$\begin{array}{ll}1 & \text { hits } \\ 2 & \text { hits }\end{array}$ 4 orgs [Coleoptera]

3 orgs

1 orgs [Tenebrionoidea; Tenebrionidae; Tribolium]

1 orgs [Chrysomeloidea; Chrysomelidae; Chrysomelinae; Timarchi: orgs [Cucujoidea; Biphyllidae; Biphyllus]

orgs [Scarabaeiformia; Scarabaeoidea; Scarabaeidae; Scarabae

orgs [Nematocera; Culicimorpha; Culicoidea; Culicidae; Anoph orgs [Pyretophorus]

2 orgs

1 orgs [Myzomyia; funestus group; funestus subgroup]

6 orgs [Brachycera; Muscomorpha; Eremoneura; Cyclorrhapha]

5 orgs

orgs [Tephritoidea; Tephritidae; Trypetinae; Carpomyini; Car

4 orgs [Ephydroidea; Drosophilidae; Drosophilinae; Drosophilin

orgs [Sophophora; obscura group]

Drosophila pseudoobscura]

1 orgs

2 orgs [repleta group; mulleri subgroup; mojavensis species col 1 orgs [virilis group]

1 orgs [Calyptratae; Oestroidea; Sarcophagidae; Sarcophaginae; orgs [Argasidae; Ornithodorinae; Ornithodoros]

orgs

1 orgs [ Ixodinae; Ixodes]

orgs

1 orgs [Dermacentor]

2 orgs [Rhipicephalus; Rhipicephalus; Rhipicephalus sanguineus

1 orgs [Aciculata; Eunicida; Amphinomidae; Eurythoe]

orgs [Brachiopoda; Phoroniformea; Phoronis]

orgs [Mollusca; Bivalvia; Pteriomorphia; Pectinoida; Pectino

orgs [Bryozoa; Gymnolaemata; Cheilostomatida; Anasca; Flust

orgs [Acoelomata; Platyhelminthes; Trematoda; Digenea; Strig orgs [Placozoa; Trichoplax]

orgs [Porifera; Demospongiae; Tetractinomorpha; Hadromerida;

3 orgs [Fungi; Dikarya; Basidiomycota; Agaricomycotina; Agari

orgs [Psathyrellaceae; Coprinopsis; Coprinopsis cinerea]

orgs [Schizophyllaceae; Schizophyllum; Schizophyllum

1 orgs [other sequences; artificial sequences]
1 orgs [Annelida/Echiura/Pogonophora group; Annelida; Polychae 


\title{
Basic Local Alignment Search Tool
}

\author{
Jump to Page Content \\ Job Title: (2) - EZ040625
}

\section{Tax BLAST Report}

Index

- Lineage Report

- Organism Repor

- Taxonomy Report

- $\underline{\text { Help }}$

Lineage Report

root
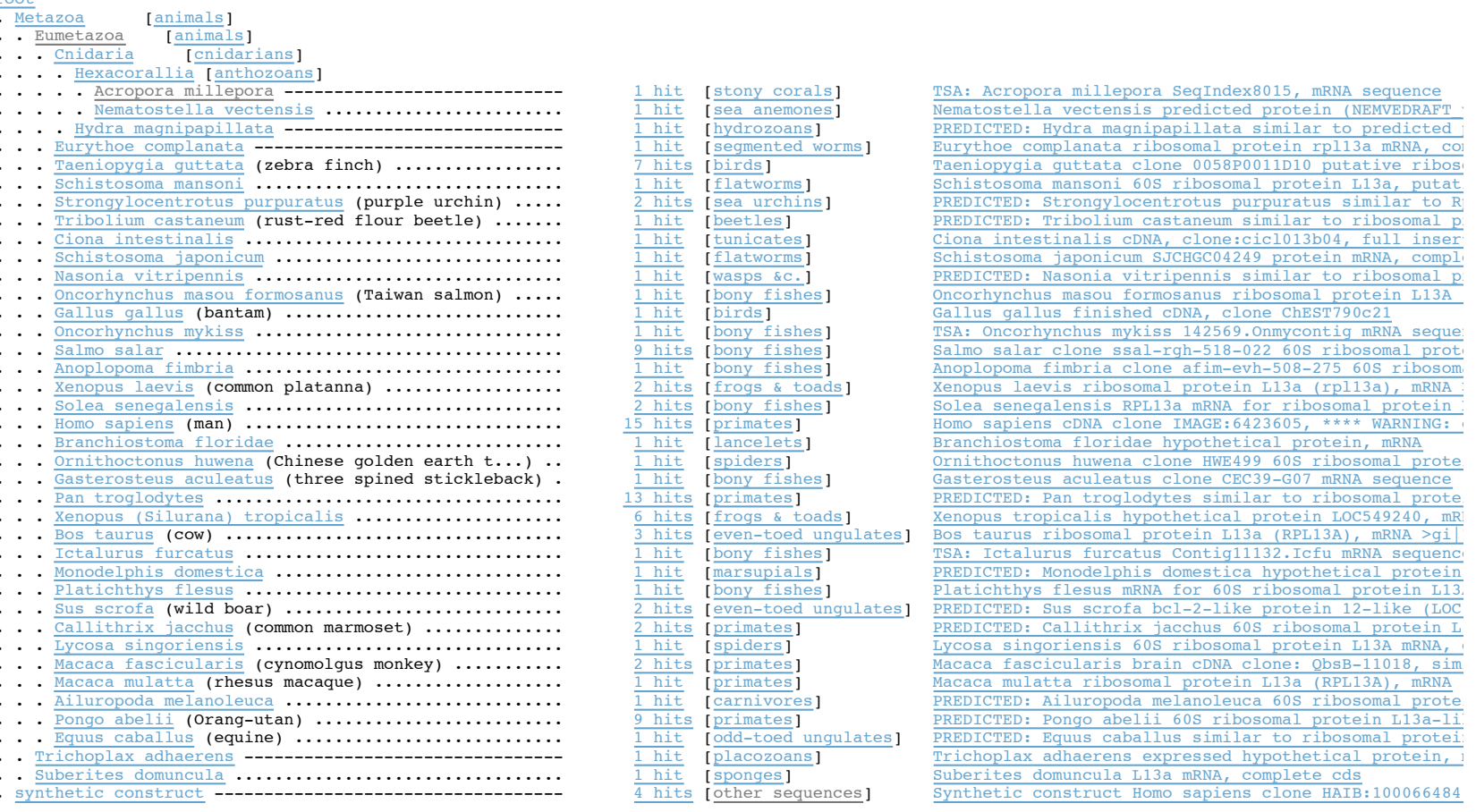

\section{Organism Report}

Acropora millepora [stony corals] taxid 45264

gb|EZ040625.1| TSA: Acropora millepora SeqIndex8015, mRNA ...

Nematostella vectensis [sea anemones] taxid 45351

ref|XM 001630762.1| Nematostella vectensis predicted prote...

Eurythoe complanata [segmented worms] taxid 167815

gb|EU125014.1| Eurythoe complanata ribosomal protein rpl13...

Taeniopygia guttata (zebra finch) [birds] taxid 59729

g|D0216005.1 | Taeniopygia guttata clone 0058P0011D10 puta...

gb DQ216004.1 Taeniopygia guttata clone 0058P0006F05 puta...

b DQ216003.1 Taeniopygia guttata clone 0058P0045E08 ribo...

0058P0016A02 puta..

.

Taeniopygia guttata clone 0058P0049C02 puta..

aerens [placozoans] taxid 10228

Trichoplax adhaerens [placozoans] taxid 10228

Schistosoma mansoni [flatworms] taxid 6183

ref|xM 002580005.1| Schistosoma mansoni 60 s ribosomal prot...

Suberites domuncula [sponges] taxid 55567

gb|AY857427.1| Suberites domuncula L13a mRNA, complete cds

Strongylocentrotus purpuratus (purple urchin, ...) [sea urchins] taxid 7668 \begin{tabular}{l|l|l|l} 
ref XM 001177209.1 & PREDICTED: Strongy locentrotus purpurat.. \\
ref XM 779422.2 PREDICTED: Strongylocentrotus purpuratus $\ldots$ & 0.0
\end{tabular}

Tribolium castaneum (rust-red flour beetle) [beetles] taxid 7070 ref|XM 969211.2| PREDICTED: Tribolium castaneum similar to...

Ciona intestinalis [tunicates] taxid 7719

dbj|AK174254.1| Ciona intestinalis cDNA, clone:cicl013b04,... 
Gallus gallus (bantam, ...) [birds] taxid 9031

emb|BX931917.1| Gallus gallus finished CDNA, clone ChEST79...

Oncorhynchus mykiss [bony fishes] taxid 8022

gb|EZ905903.1| TSA: Oncorhynchus mykiss 142569. onmycontig ...

Salmo salar [bony fishes] taxid 8030

Salmo salar clone ssal-rgh-518-022 60s ribo...

Salmo salar clone ssal-rgh-518-022 605 ribo.

Salmo salar clone ssal-rgh-518-022 60 ribo...

Salmo salar clone sal-rghe

Salmo salar clone Contig1152 60s ribosomal ...

Salmo salar clone Contig2288 60S ribosomal ....

Salmo salar clone ssal-eve-575-212 60 s ribo...

Salmo salar clone HM6 0617 ribosomal protei...

gb BT044023.1 Salmo salar clone HM6_0789 ribosomal protei...

Anoplopoma fimbria [bony fishes] taxid 229290

gb|BT082277.1| Anoplopoma fimbria clone afim-evh-508-275 6...

Xenopus laevis (common platanna, ...) [ frogs \& toads] taxid 8355 gb $\mathrm{BC} 043976.1$ Xenopus

Solea senegalensis [bony fishes] taxid 28829

bj|AB360597.1 Solea senegalens is RPL13a mRNA for ribosom... dbj $A B 374949.1$ Solea senegalensis RPL13a-likel or ribosom...

Homo sapiens (man) [primates] taxid 9606

gb|BC071921.1 Homo sapiens CDNA clone IMAGE: $6423605, * * * * \ldots$

gb $\mathrm{BC} 062537.1$ Homo sapiens CDNA clone IMAGE:6423605, $* * \star \star .$.

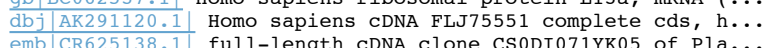
full-length CDNA Clone CSODI071YK05 of Pla... full-length CDNA clone CSODE004YD03 of Pla... full-length CDNA clone CSODL002YH12 of B C... full-length CDNA clone CS0DI069YJ19 of Pla... full-length CDNA clone CSODI075YJ21 of Pla... full-length CDNA clone CSODE001YC16 of Pla... full-length CDNA clone CSODI058YC16 of Pla.. full-length CDNA clone CSODD008YF03 of Neu... full-length CDNA clone CSODC008YA18 of Neu... full-length CDNA clone CSODI065YB06 of Pla...

Xenopus (Silurana) tropicalis [frogs \& toads] taxid 8364 gb|BC168117.1| Xenopus tropicalis hypothetical protein LOC... gb BC168111.1 Xenopus tropicalis hypothetical protein LOC... gb BC160997.1 Xenopus tropicalis hypothetical protein LOC... gb BC135476.1 Xenopus tropicalis hypothetical protein LOC..

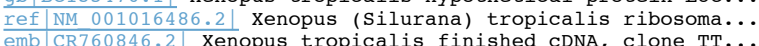

Bos taurus (cow, ...) [even-toed ungulates] taxid 9913 ref|NM 001076998.1 Bos taurus ribosomal protein L13a (RPL... gb $B C 103039.1 \mid$ Bos taurus ribosomal protein L13a, mRNA (CD... emb|x56933.1| B.taurus mRNA, alternative polyadenylation s..

Ictalurus furcatus [bony fishes] taxid 66913 gb|HP440486.1| TSA: Ictalurus furcatus Contig11132.Icfu mR... Monodelphis domestica [marsupials] taxid 13616 ref|XM 001363184.1| PREDICTED: Monodelphis domestica hypot... platichthys flesus [bony fishes] taxid 8260 emb|AJ843089.1| Platichthys flesus mRNA for 60 s ribosomal ...

Sus scrofa (wild boar, ...) [even-toed ungulates] taxid 9823 

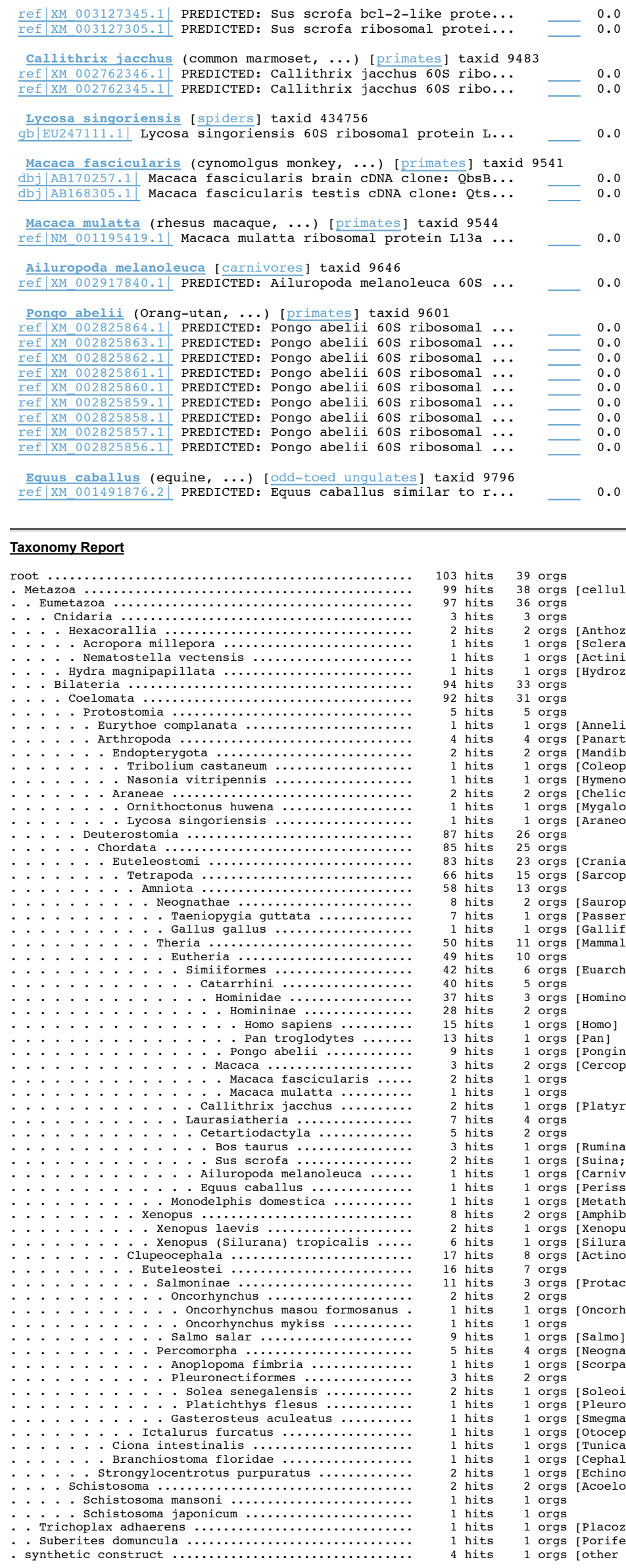

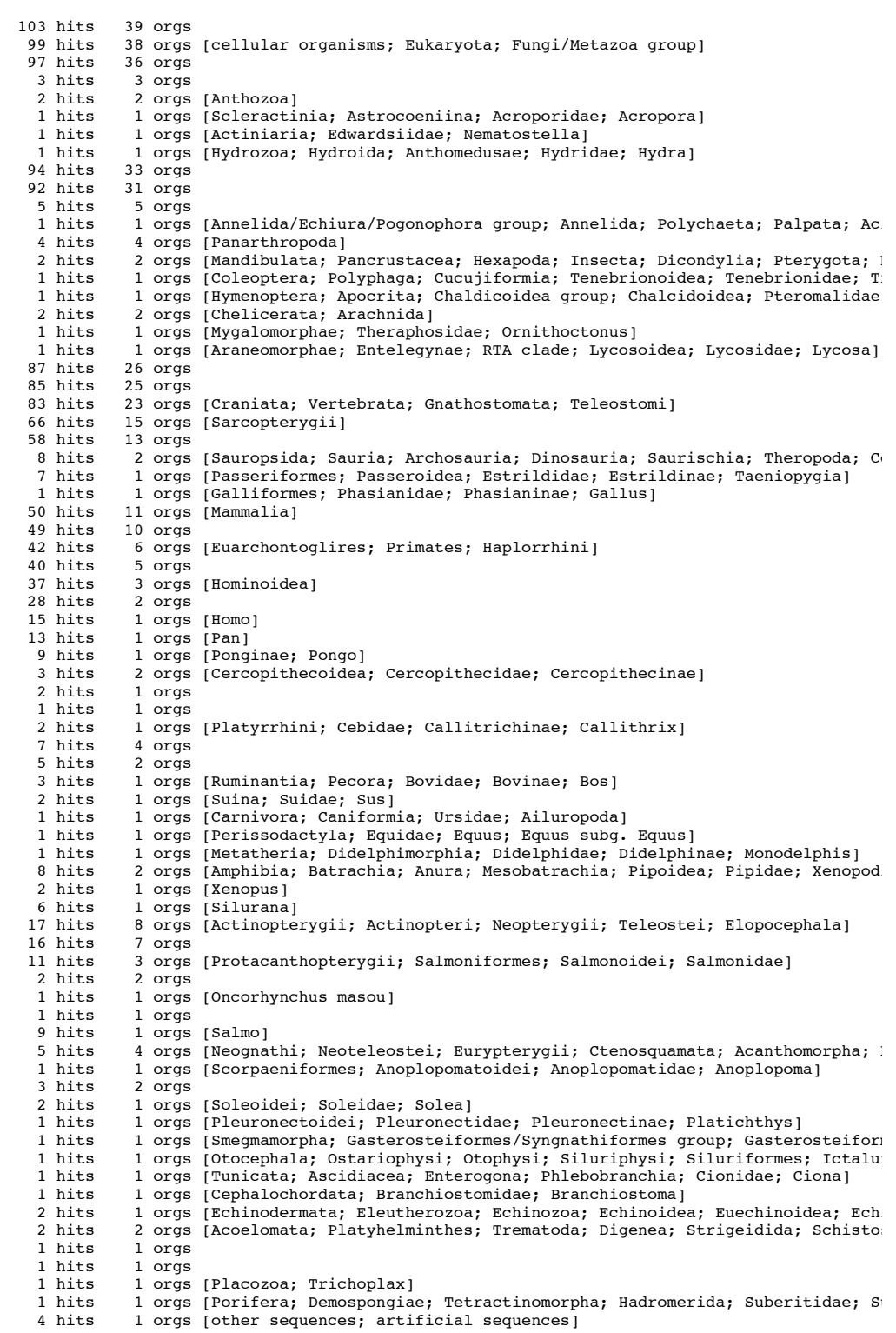




\section{Basic Local Alignment Search Tool}

Jump to Page Content

\section{Tax BLAST Report}

Index

- Lineage Report

- Organism Repor

- Taxonomy Repor

- $\underline{\text { Help }}$

\section{Lineage Report}

root
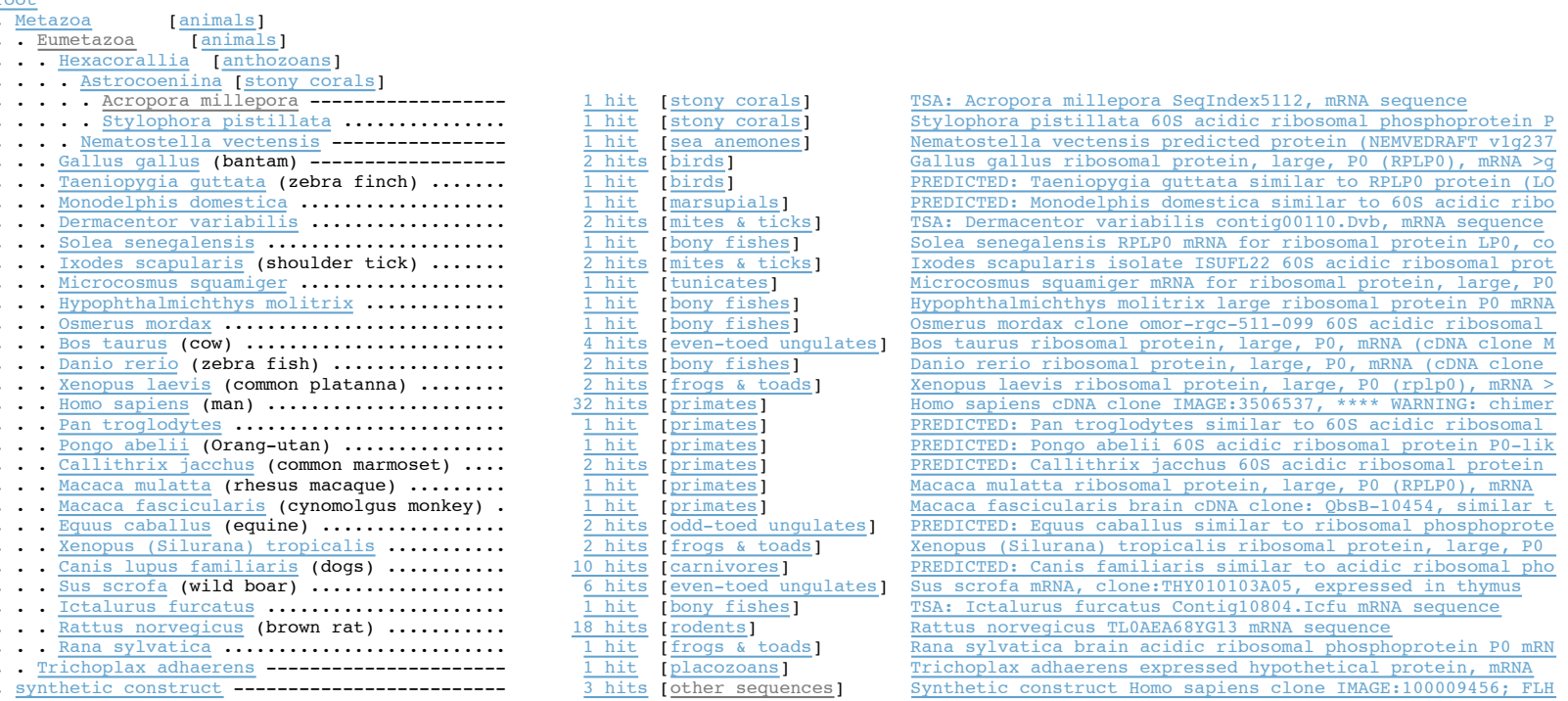

\section{Organism Report}

Acropora millepora [stony corals] taxid 45264

gb|Ez028666.1| TSA: Acropora millepora SeqIndex5112, mRNA ... 0.0

Stylophora pistillata [stony corals] taxid 50429

gb|EU069460.1| Stylophora pistillata 60 s acidic ribosomal ...

Nematostella vectensis [sea anemones] taxid 45351

ref|XM 001626244.1| Nematostella vectensis predicted prote...

Gallus gallus (bantam, ...) [birds] taxid 9031

ef NM 204987.1 Gallus gallus ribosomal protein, large, P...

gb $\mathrm{L} 28704.1 \mid$ CHKACRIPHO Chicken acidic ribosomal phosphopro...

Taeniopygia guttata (zebra finch) [birds] taxid 59729

ref|xM 002199525.1| PREDICTED: Taeniopygia guttata similar...

Monodelphis domestica [marsupials] taxid 13616

ref|XM_001363500.1| PREDICTED: Monodelphis domestica simil...

Dermacentor variabilis [mites \& ticks] taxid 34621

|F7525048.1 TSA: Dermacentor variabilis contig00110. Dvb...

gb EZ532706.1 TSA: Dermacentor variabilis contig00067.Dvc...

Solea senegalensis [bony fishes] taxid 28829

dbj|AB374983.1| Solea senegalensis RPLP0 mRNA for ribosoma...

Ixodes scapularis (shoulder tick, ...) [mites \& ticks] taxid 6945 gb|DQ066213.1| Ixodes scapularis isolate ISUFL22 60S acidi... ref|XM_002399569.1| Ixodes scapularis 60S acidic ribosomal...

Microcosmus squamiger [tunicates] taxid 439822

emb|FN984781.1| Microcosmus squamiger mRNA for ribosomal p...

Hypophthalmichthys molitrix [bony fishes] taxid 13095

gb|HM124745.1| Hypophthalmichthys molitrix large ribosomal...

Osmerus mordax [bony fishes] taxid 8014

gb|BT074646.1| osmerus mordax clone omor-rgc-511-099 60s a...

Bos taurus (cow, ...) [even-toed ungulates] taxid 9913

gb BC151695.1 Bos taurus ribosomal protein, large, P0, mR...

gb $\mathrm{BC1} 102074.1$ Bos taurus ribosomal protein, large, P0, mR..

gb

Danio rerio (zebra fish, ...) [bony fishes] taxid 7955 
Pan troglodytes [primates] taxid 9598

Pongo abelii (Orang-utan, ...) [primates] taxid 9601

ref|XM 002823848.1| PREDICTED: Pongo abelii 60s acidic rib..

Macaca mulatta (rhesus macaque, ...) [primates] taxid 9544

Equus caballus (equine, ...) [odd-toed ungulates] taxid 9796 $\begin{array}{llll}\text { ref } & \text { XM } 001489353.1 & \text { PREDICTED: Equus caballus similar to } r . . . \\ \text { ref XM } 001489330.1 & \text { PREDICTED: Equus caballus similar to } r . .\end{array}$

Xenopus (Silurana) tropicalis [frogs \& toads] taxid 8364 ref|NM 203736.1| Xenopus (Silurana) tropicalis ribosomal p...

Canis lupus familiaris (dogs) [carnivores] taxid 9615

ef|XM 857926.1| PREDICTED: Canis familiaris similar to ac... ref XM 857980.1 PREDICTED: Canis familiaris similar to ac... ref XM 844092.1 PREDICTED: Canis familiaris ribosomal pro... ref XM 858027.1 PREDICTED: Canis familiaris similar to ac... ref XM 858052.1 PREDICTED: Canis familiaris similar to ac... ref XM 858004.1 PREDICTED: Canis familiaris similar to ac... ref XM 535894.2 PREDICTED: Canis familiaris ribosomal pro... PREDICTED: Canis familiaris ribos PREDICTED: Canis familiaris ribosomal pro... ref XM 845901.1 PREDICTED: Canis familiaris similar to ac... Sus scrofa (wild boar, ....) [even-toed ungulates] taxid 9823 dbj|AK239428.1| Sus scrofa mRNA, clone:THY010103A05, expre... \begin{tabular}{l|l|l|l} 
ref NM 001098598.1 & Sus scrofa ribosomal phosphoprotein la... \\
\hline gb DO316319.1 Sus scrofa ribosomal phosphoprotein large P...
\end{tabular} Sus scrofa mRNA, clone: OVR010082B07, expre... \begin{tabular}{l|l}
$\mathrm{dbj} A K 231505.1$ & Sus scrofa mRNA, clone:ITT010083C10, expre... \\
\hline $\mathrm{dbjAK234557.1}$ Sus scrofa mRNA, clone:OVR010084G10, expre..
\end{tabular}

Ictalurus furcatus [bony fishes] taxid 66913 gb|HP440171.1| TSA: Ictalurus furcatus Contig10804.Icfu mR...

Rattus norvegicus (brown rat, ...) [rodents] taxid 10116 emb|FQ231733.1| Rattus norvegicus TL0AEA68YG13 mRNA sequence emb FQ231525.1 Rattus norvegicus TL0AEA6YC21 mRNA sequence \begin{tabular}{l|l} 
emb FQ229397.1 Rattus norvegicus TLOADA48YB05 mRNA sequence \\
\hline emb FO220533.1
\end{tabular} emb FQ220533.1 Rattus norvegicus TLOADA42YI10 mRNA sequence emb FO225860.1 Rattus norvegicus TLOAEA3YO11 mRNA sequence Rattus norvegicus TL0AEA68YP07 mRNA sequence Rattus norvegicus TLOADA42YH23 mRNA sequence Rattus norvegicus TLOADA42YI17 mRNA sequence Rattus norvegicus TLOADA43YH18 mRNA sequence Rattus norvegicus TLOADA44YI12 mRNA sequence Rattus norvegicus TL0AAA50YK12 mRNA sequence Rattus norvegicus TL0ADA48YA13 mRNA sequence Rattus norvegicus TLOADA48YH 23 mRNA sequence Rattus norvegicus TLOADA4YM19 mRNA sequence
Rattus norvegicus TLOAEA67YI08 mRNA sequence 
attus norvegicus TLOACA52YN20 mRNA sequence

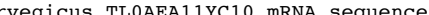
Rattus norvegicus TL0AEA62YC06 mRNA sequence

Rana sylvatica [frogs \& toads] taxid 45438

gb|AF176302.1| Rana sylvatica brain acidic ribosomal phosp...

Trichoplax adhaerens [placozoans] taxid 10228

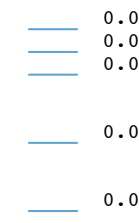

\section{Taxonomy Report}

root

Metazoa ...

. Eumetazoa...

. Astrocoeniina

. . Acropora millepor

.. Stylophora pistillat

$\therefore$. Coelomata

$\therefore$ Chordata .......

. . . Tetrapoda

. . . . . Amniota . Neognathae

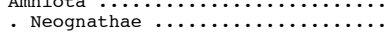

$\therefore$ Taeniopygia guttat

$\therefore$. . . Theria $\ldots \ldots$ Monodelphis domestica

.. Eutheria

................

$\therefore \therefore$ Laurasiatheria

....... Bos taurus

. . . Sus scrofa

... . . . Canis lupus familiaris ...

..... Euarchontoglire

. . Eatarrini

.......

. . . Homo sapiens . ........

$\ldots \ldots . . .$. Pan troglodytes ...

Macaca

. Macaca..............

. . . Macaca fascicularis

. . . . . Callithrix jacchus .....

. . Xenopus

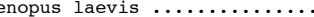
Xenopus (Silurana) tropicalis - Xenopus (Silurana) tropicalis clupeocephala

. . . Euteleostei

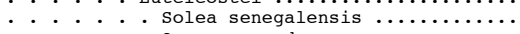

$\therefore$ Otophysi

. . . . Cyprinidae $\ldots \ldots \ldots \ldots \ldots \ldots$ (n.............

Microcosmus squamiger

Dermacentor variabilis

Dermacentor variabi

- Trichodes scapular

synthetic construct
105 hits 30 orgs

102 hits 29 orgs [cellular organisms; Eukaryota; Fungi/Metazoa group]

101 hits 28 orgs

3 hits 3 orgs [Cnidaria; Anthozoa]

2 hits 2 orgs [Scleractinia]

hits 1 orgs [Acroporidae; Acropora]

1 orgs [Pocilloporidae; Stylophora]

$\begin{aligned} 1 & \text { hits } 1 \text { orgs [Actiniaria, } \\ 98 & \text { hits } 25 \text { orgs [Bilateria] }\end{aligned}$

94 hits 23 orgs [Deuterostomia]

93 hits 22 orgs [Craniata; Vertebrata; Gnathostomata; Teleostomi]

87 hits 17 orgs [Sarcopterygii]

82 hits 14 orgs 3 orgs [Sauropsida; Sauria; Archosauria; Dinosauria; Saurischia; Theropoda; Coeluro

hits 1 orgs [Galliformes

Passeroidea; Estrildidae; Estrildinae; Taeniopygia]

12 orgs [Mannalia]

1 (Midelphimorphia; Didelphidae; Didelphinae; Monodelphis

78 hits 11 orgs

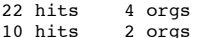

4 hits 1 orgs [Ruminantia; Pecora; Bovidae; Bovinae; Bos]

6 hits 1 orgs [Suina; Suidae; Sus]

2 hits 1 orgs [Perissodactyla; Equidae; Equus; Equus subg. Equus]

10 hits 1 orgs [Carnivora; Caniformia; Canidae; Canis; Canis lupus]

56 hits 7 orgs

6 orgs [Primates; Haplorrhini]

36 hits 5 orgs

34 hits 3 orgs [Hominoidea]

32 hits 1 orgs [Homo]

1 hits 1 orgs [Pan]

1 hits 1 orgs [Ponginae; Pongo]

2 hits 2 orgs [Cercopithecoidea; Cercopithecidae; Cercopithecinae]

1 hits 1 orgs

hits

2 hits

5 hits

hits

2 hits

1 hits

6 hits

2 hits

1 hits

1 hits

4 hits

1 hits

2 hits

2 hits

1 hits

4 hits

2 hits

2 hits

1 hits

1 orgs [Platyrrhini; Cebidae; Callitrichinae; Callithrix]
1 orgs [Glires; Rodentia; Sciurognathi; Muroidea; Muridae; Murinae; Rattus]

orgs [Amphibia; Batrachia]

orgs [Mesobatrachia; Pipoidea; Pipidae; Xenopodinae]

1 orgs [Xenopus]

1 orgs [Neobatrachia; Ranoidea; Ranidae; Raninae; Rana; Novirana; Aquarana/sylvatic 5 orgs [Actinopterygii; Actinopteri; Neopterygii; Teleostei; Elopocephala]

1 orgs [Neognathi; Neoteleostei; Eurypterygii; Ctenosquamata; Acanthomorpha; Euacan 1 orgs [Protacanthopterygii; Osmer

3 orgs [Otocephala; Ostariophysi]
2 orgs [Cypriniphysi; Cypriniformes; Cyprinoidea]
1 orgs [Hypophthalmichthys]

1 orgs [Danio]

orgs [Siluriphysi; Siluriformes; Ictaluridae; Ictalurus]

1 orgs [Tunicata; Ascidiacea; Stolidobranchia; Pyuridae; Microcosmus]

2 orgs [Protostomia; Panarthropoda; Arthropoda; Chelicerata; Arachnida; Acari; Para

1 orgs [Rhipicephalinae;

orgs [Ixodinae; Ixodes]

1 orgs [other sequences; artificial sequences] 


\section{Basic Local Alignment Search Tool}

Jump to Page Content

\section{Tax BLAST Report}

Index

- Lineage Report

- Organism Report

- Taxonomy Repor

- $\underline{\text { Help }}$

\section{Lineage Report}

root

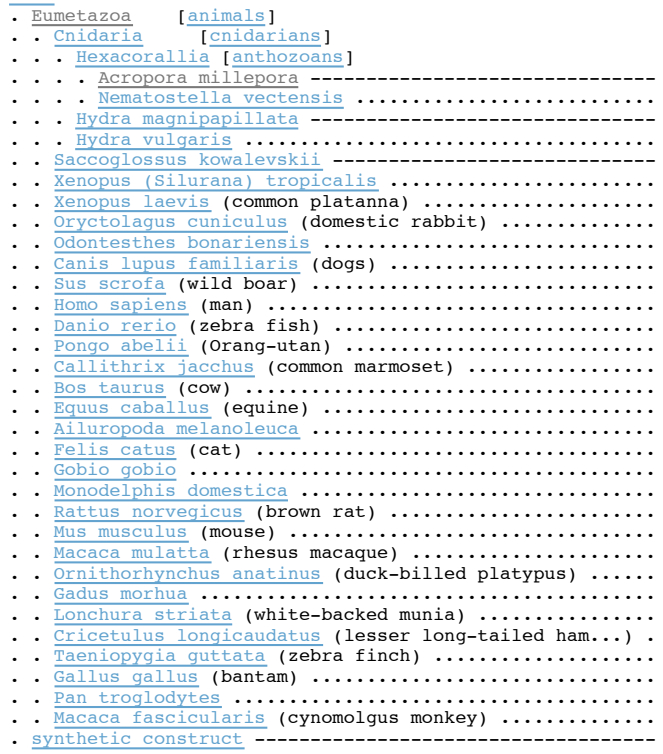

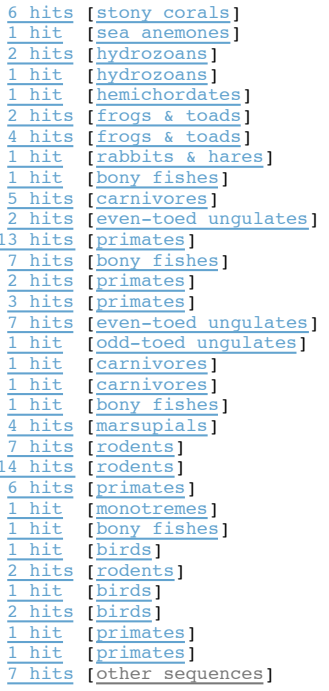

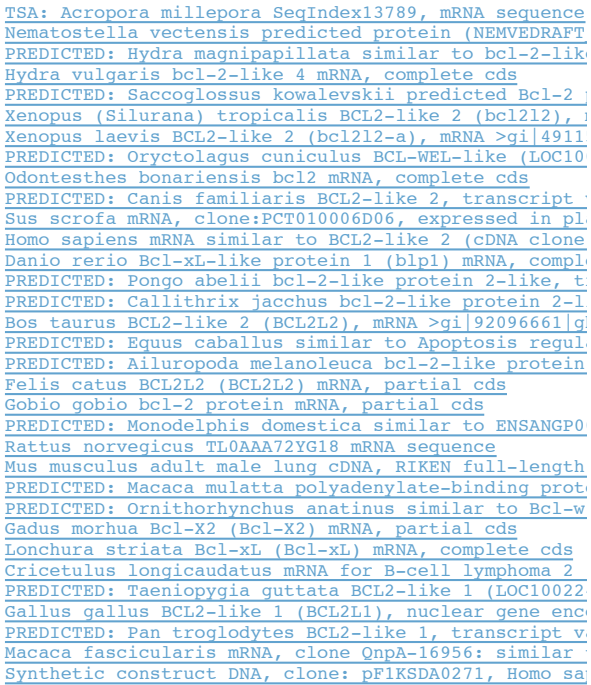

\section{Organism Report}

Acropora millepora [stony corals] taxid 45264

b|EZ011917,1| TSA: Acropora millepora SeqIndex13789, mRNA...

b EU161957.1 Acropora millepora Bcl-like protein mRNA, c...

g

gb

gb EZ011916.1 TSA: Acropora millepora SeqIndex13788, mRNA...

Nematostella vectensis [sea anemones] taxid 45351

ef|XM 001634647.1| Nematostella vectensis predicted prote...

Hydra magnipapillata [hydrozoans] taxid 6085

(2085illata simila..

\begin{tabular}{l|l|l|l} 
ref XM 002167542.1 & PREDICTED: Hydra magnipapillata simila... \\
\hline
\end{tabular}

Hydra vulgaris [hydrozoans] taxid 6087

gb|EU035764.1| Hydra vulgaris bcl-2-like 4 mRNA, complete cds

Saccoglossus kowalevskii [hemichordates] taxid 10224

ref|XM 002740743.1| PREDICTED: Saccoglossus kowalevskii pr...

Xenopus (Silurana) tropicalis [frogs \& toads] taxid 8364

( gb BCO 84445.1 Xenopus tropicalis Bcl2-like 2, mRNA (CDNA ...

Xenopus laevis (common platanna, ...) [frogs \& toads] taxid 8355

I 001092260.1 Xenopus laevis BCL2-like 2 (bcl212-a),... gb BC073259.1 Xenopus laevis MGC80617 protein, mRNA (CDNA.. ref NM 001088104.1 Xenopus laevis BCL2-like 2 (bcl212-b) ,...

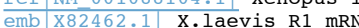
Oryctolagus cuniculus (domestic rabbit, ...) [rabbits \& hares] taxid 9986
ref|XM 002717903.1| PREDICTED: Oryctolagus cuniculus BCL-W...

Odontesthes bonariensis [bony fishes] taxid 219752

gb|FJ868843.1| Odontesthes bonariensis bcl2 mRNA, complete...

Canis lupus familiaris (dogs) [carnivores] taxid 9615

ref XM 853671.1| PREDICTED: Canis familiaris BCL2-like 2, ... ref XM 853783.1 PREDICTED: Canis familiaris BCL2-like 2, ... ef|NM $001031635.2 \mid$ Canis lupus familiaris poly(A) binding... gb DQ116955.1 Canis familiaris Bcl-w protein (BCL2L2) mRN.

AY509563.1 Canis familiaris Bcl-2 mRNA, complete cds

Sus scrofa (wild boar, ...) [even-toed ungulates] taxid 9823

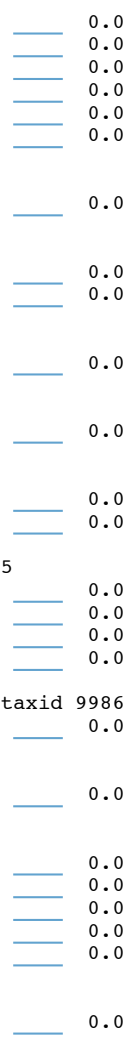




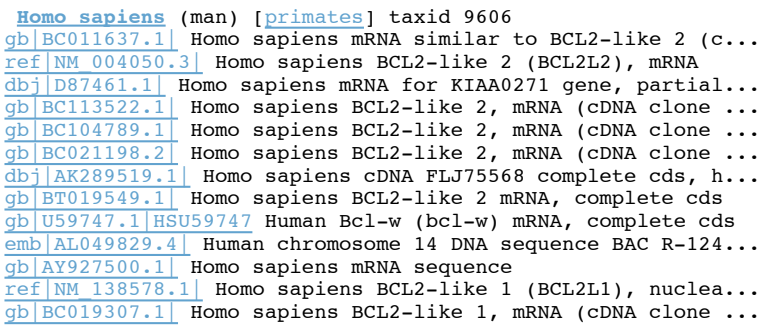

gb $\mathrm{BC} 019307.1$ Homo sapiens BCL2-like 1, mRNA (CDNA clone...

Danio rerio (zebra fish, ...) [bony fishes] taxid 7955

gb DQ022560.1 1 Danio rerio Bcl-xL-like protein 1 (blp1) mR... (bef 131807 1 Danio rorio bcl2-like 1 (bcl211), nuclear... gb AF317837.1 AF 317837 Danio rerio Bcl-XL-like protein 1 (... gb BC165156.1 Danio rerio bc12-11ke, mRNA (CDNA Clone MGC... ref XM 001341178,2l PREDICTED: Danio rerio BCl2-like (LOC1...

Pongo abelii (Orang-utan, ...) [primates] taxid 9601 ef XM 002824582.1 PREDICTED: Pongo abelii bcl-2-like pro... ref XM 002824583.1 PREDICTED: Pongo abelii bal-2-like pro...

Callithrix jacchus (common marmoset, ...) [primates] taxid 9483 PREDICTED: Callithrix jacchus bcl-2-li... ref XM 002747332.1 PREDICTED: Callithrix jacchus bcl-2-li... PREDICTED: Callithrix jacchus bcl-2-li... Bos taurus (cow, ...) [even-toed ungulates] taxid 9913 gb BC114652, Bos taurus BCL2-like 2, mRNA (CDNA clone MG... $\mathrm{dbj|AB238938.1|}$ Bos taurus BCL2L2 mRNA for BCL2-like 2, co... gb DQ001758.1 Bos taurus BCL2-like protein 2 (BCL2L2) gen... gb BC147863.1 Bos taurus BCL2-like 1, mRNA (CDNA clone MG... gb BC133281.1 Bos taurus BCL2-like 1, mRNA (CDNA clone MG...

Equus caballus (equine, ...) [odd-toed ungulates] taxid 9796 ref|XM 001493694.2 | PREDICTED: Equus caballus similar to A...

Ailuropoda melanoleuca [Carnivores] taxid 9646 ref|XM 002926013.1| PREDICTED: Ailuropoda melanoleuca bcl-...

Felis catus (cat, ...) [carnivores] taxid 9685 gb|DQ926874.1 Felis catus BCL2L2 (BCL2L2) mRNA, partial cds

synthetic construct [other sequences] taxid 32630 dbj|AB383828.1| Synthetic construct DNA, clone: pF1KSDA027... gb BT019548,1 Synthetic construct Homo sapiens BCL2-like... gb AY89081hetic construct Homo sapiens clone FLH0 ... gb AY890433.1 Synthetic construct Homo sapiens clone FLH1... gb AY892894.1 Synthetic construct Homo sapiens clone FLH0... gb AY888228.1 Synthetic construct Homo sapiens clone FLHO..

Gobio gobio [bony fishes] taxid 27704

gb|FJ612582.1 Gobio gobio bcl-2 protein mRNA, partial cds

Monodelphis domestica [marsupials] taxid 13616

ref|XM 001369257.1 PREDICTED: Monodelphis domestica simil... ref XM 001365223.1 PREDICTED: Monodelphis domestica simil... ref XM 001365293.1 PREDICTED: Monodelphis domestica simil...

Rattus norvegicus (brown rat, ...) [rodents] taxid 10116

emb|FO212018.1| Rattus norvegicus TL0AAA72YG18 mRNA sequence ref|NM 021850.2 Rattus norvegicus Bcl2-like 2 (Bcl212), mRNA gb $\mathrm{BC} 074021$ 1 Rattus norvegicus Bcl2-like 2, mRNA (CDNA c.. gb AY185098.1 Rattus norvegicus BCL-W mRNA, complete cds gb AY185100.1 Rattus norvegicus BCL-WEL mRNA, complete cds

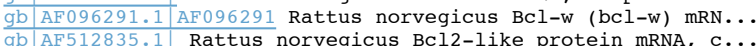

\section{Mus musculus (mouse) [ rodents] taxid 10090}

dbj|AK004680.1 Mus musculus adult male lung cDNA, RIKEN f... ref NM 007537.1| Mus musculus BCL2-like 2 (Bc1212), mRNA gb AF030769.1 $A F 030769$ Mus musculus BCL-W (BCl-w) mRNA, Co... \begin{tabular}{l|l|l}
$\mathrm{dbj}$ AK172925.1 & Mus musculus mRNA for mKIAA0271 protein \\
\hline $\mathrm{dbj}$ & MK015644.1 & Mus \\
&
\end{tabular} dbjAK015644.1 Mus musculus adult male testis CDNA, RIKEN... gb U59746.1 MMU59746 Mus musculus Bcl-w (bcl-w) mRNA, comp.. gb AC116591.4 Mus musculus BAC clone RP24-90K1 from chrom... emb $\mid$ CT025533.11 $\mid$ Mouse DNA sequence from clone RP23-93K3 o... gb $\mathrm{BC} 040369.1$ Mus musculus BCL2-like 2, mRNA (CDNA clone... dbj AK013244.1 Mus musculus 10, 11 days embryo whole body... fef $\mid$ NM $009741.3 \mid$ Mus musculus B-cell leukemia/lymphoma 2 (...

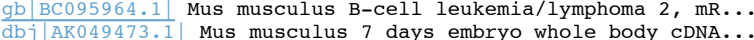
dbjAK172250.1 Mus musculus activated spleen CDNA, RIKEN .. \begin{tabular}{l} 
Macaca mulatta (rhesus macaque, ...) [primates] taxid 9544 \\
ref XM 001107287.2 \\
\hline
\end{tabular} ref XM 001110326.1 PREDICTTED: Macaca mulatta bcl-2-like p... PREDICTED: Macaca mulatta bcl-2-like p... PREDICTED: Macaca mulatta bcl-2-like p... PREDICTED: Macaca mulatta bcl-2-like p... PREDICTED: Macaca mulatta bcl-2-like p...
PREDICTED: Macaca mulatta bcl-2-like p... 

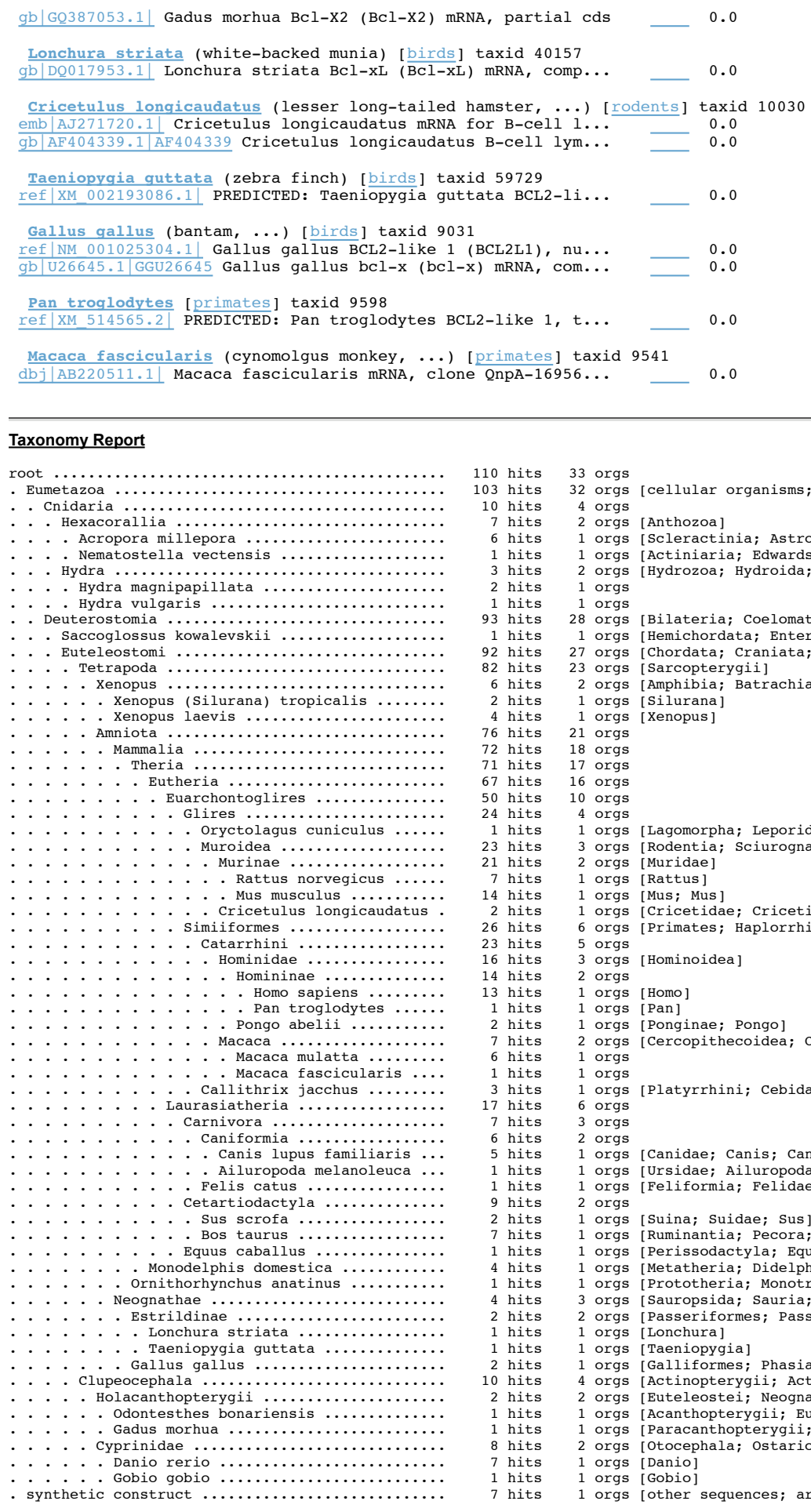

110 hits 33 org

103 hits 32 orgs [cellular organisms; Eukaryota; Fungi/Metazoa group; Metazoa]

10 hits 4 org

7 hits 2 orgs [Anthozoa]
6 hits 1 orgs [Scleractinia; Astrocoeniina; Acroporidae; Acropora]
1 hits 1 orgs [Actiniaria; Edwardsiidae; Nematostella]

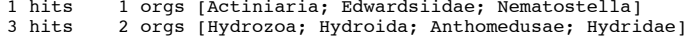

2 hits 1 orgs

1 hits

93 hits

92 hits

82 hits

6 hits

2 hits

4 hits

72 hits

71 hits

67 hits

50 hits
24 hits

24 hits
1 hits

23 hits

21 hits
7 hits

14 hits

14 hits
2 hits

26
hits

16 hits

14 hits

13 hits

1 hits

2 hits

7 hits

6 hits

3 hits
17 hits

7 hits

6 hits

5 hits

1 hits

1 hits

9 hits

2 hits

$\begin{array}{ll}7 & \text { hits } \\ 1 & \text { hits }\end{array}$

4 hits

1 hits

4 hits

2 hits

1 hits

1 hits

2 hits

10 hits

2 hits

1 hits

8 hits

7 hits

1 hits
7 hits

$\begin{aligned} 1 & \text { orgs } \\ 28 & \text { orgs [Bilateria; Coelomata] }\end{aligned}$

1 orgs [Hemichordata; Enteropneusta; Harrimaniidae; Saccoglossus]

27 orgs [Chordata; Craniata; Vertebrata; Gnathostomata; Teleostomi

3 orgs [Sarcopterygii]

orgs [Amphibia; Batrachia; Anura; Mesobatrachia; Pipoidea; Pipidae; Xenopodinae] orgs [Silurana]

21 orgs

18 orgs

6 orgs

0 orgs

4 org

1 orgs [Lagomorpha; Leporidae; Oryctolagus]

3 orgs [Rodentia; Sciurognathi]

2 orgs [Muridae]

orgs [Rattus]

orgs [Cricetidae; Cricetinae; Cricetulus]

6 orgs [Primates; Haplorrhini]

5 orgs

3 orgs [Hominoidea]

2 orgs

1 orgs [Homo]

1 orgs [Pan]

orgs [Ponginae; Pongo]

orgs [Cercopithecoidea; Cercopithecidae; Cercopithecinae]

1 orgs

1 orgs [Platyrrhini; Cebidae; Callitrichinae; Callithrix]

6 orgs

3 orgs

1 orgs [Canidae; Canis; Canis lupus]

1 orgs [Ursidae; Ailuropoda]

1 orgs [Feliformia; Felidae; Felinae; Felis]

orgs [Suina; Suidae; Sus]

orgs [Ruminantia; Pecora; Bovidae; Bovinae; Bos]

; Equus; Equus subg. Equus

dae; Didelphinae; Monodelphis

orgs Prototheria; Monotremata; Ornithorhynchidae; Ornithorhynchus]

3 orgs [Sauropsida; Sauria; Archosauria; Dinosauria; Saurischia; Theropoda; Coelur orgs [Passeriformes; Passeroidea; Estrildidae]

1 orgs [Galliformes; Phasianidae; Phasianinae; Gallus]

4 orgs [Actinopterygii; Actinopteri; Neopterygii; Teleostei; Elopocephala]

orgs [Euteleostei; Neognathi; Neoteleostei; Eurypterygii; Ctenosquamata; Acantho

[Acanthopterygii; Euacanthopterygii; Percomorpha; Smegmamorpha; Atherinomor

[Paracanthopterygil; Gadiformes; Gadidae; Gadus

1 orgs [Danio]

1 orgs [Gobio]

1 orgs [other sequences; artificial sequences] 


\section{Basic Local Alignment Search Tool}

Jump to Page Content

Job Title: (2) - EZ037140

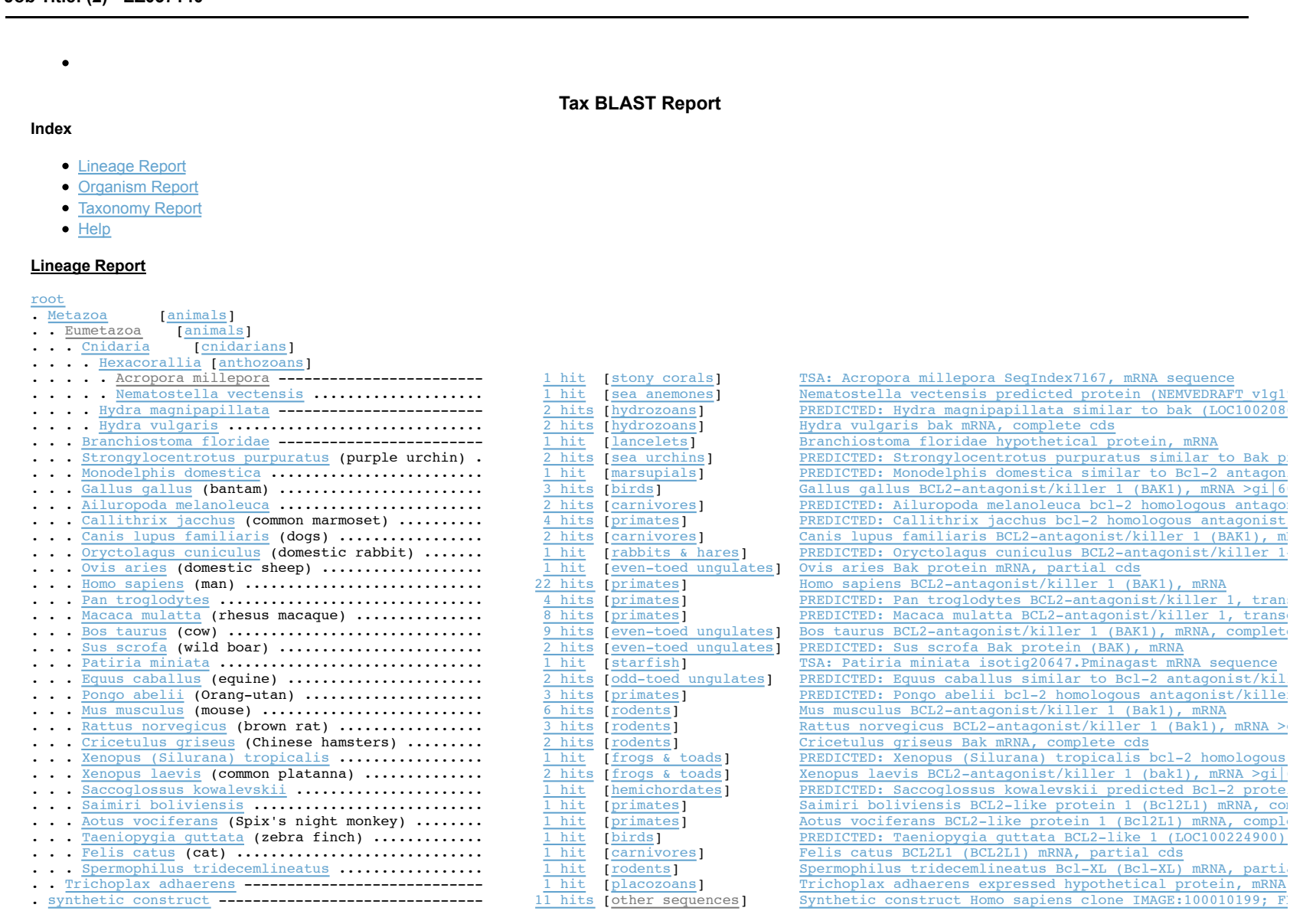

\section{Organism Report}

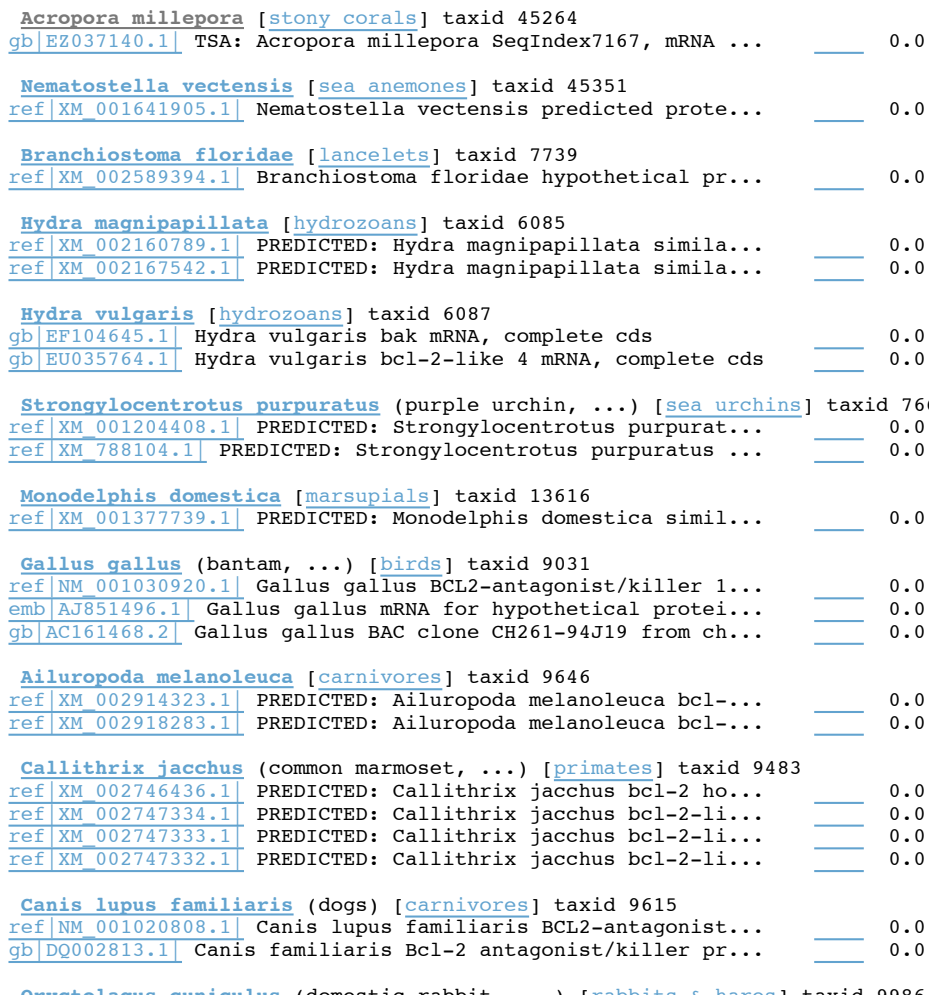


Macaca mulatta (rhesus macaque, ...) [primates] taxid 9544
ref XM 001090570.2
PREDICTED: Macaca mulatta BCL2-antagon...

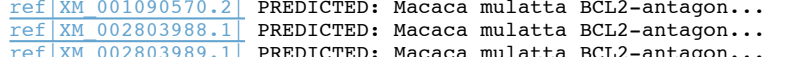
ef XM 002803989.1 PREDICTED: Macaca mulatta BCL2-antagon... PREDICTED: Macaca mulatta bcl-2-like p... PREDICTED: Macaca mulatta bcl-2-like p... PREDICTED: Macaca mulatta bcl-2-like p... PREDICTED: Macaca mulatta bcl-2-like p...

\begin{tabular}{l|l} 
Synthetic construct [other sequences] taxid 32630 \\
gb|Do895739.2 & Synthetic construct Homo sapiens clone IMAG...
\end{tabular} gb DQ892527.2 Synthetic construct clone IMAGE: $100005157 ; \ldots$ gb AY893853.1 Synthetic construct DNA, clone: pFN21AB694... gb AY893403.1 Synthetic construct Homo sapiens clone FLHO... $\frac{\mathrm{gbA} A Y 889585.1}{\mathrm{dbj} A B 528577.1}$ Synthetic construct Homo sapiens clone FLH1... Synthetic construct Homo sapiens gateway c...

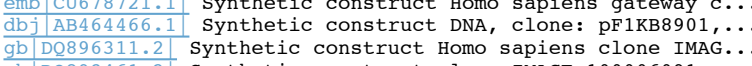
gb DQ893461.2 Synthetic construct clone IMAGE: $100006091 ; \ldots$

Bos taurus (cow, ...) [even-toed ungulates] taxid 9913 gb|BT030701.1 Bos taurus BCL2-antagonist/killer 1 (BAK1),... gb BC146049. Bos taurus BCL2-antagonist/killer 1, mRNA $(. .$. ref $\mid$ NM $001077918.1 \mid$ Bos taurus BCL2-antagonist/killer 1 (B... $\mathrm{dbj}|\mathrm{AB} 238939.1|$ Bos taurus BAK1 mRNA for Bcl-2 homologous ... gb HM630302.1 Bos taurus BCL2-like protein 1 (BCl2L1) mRN... gb BC147863.1 Bos taurus BCL2-like 1, mRNA (CDNA clone MG...

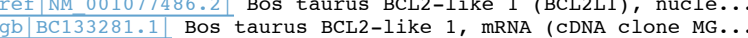
dbj $|\mathrm{AB} 238936.1|$ Bos taurus BCL2L1 mRNA for BCL2-like 1 tra...

Sus scrofa (wild boar, ...) [even-toed ungulates] taxid 9823 ef XM 001928147.2 | PREDICTED: Sus scrofa Bak protein (BAK...

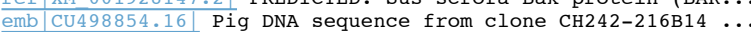

Patiria miniata [starfish] taxid 46514

gb|HP125449.1| TSA: Patiria miniata isotig20647.Pminagast ...

0.

Equus caballus (equine, ...) [odd-toed ungulates] taxid 9796

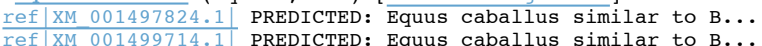

Pongo abelii (Orang-utan, ...) [primates] taxid 9601

ref|XM 002824315.1| PREDICTED: Pongo abelii bcl-2 homologo... ref XM 002816764.1 PREDICTED: Pongo abelii bcl-2 homologo... ref XM 002830190.1 PREDICTED: Pongo abelii bcl-2-like pro...

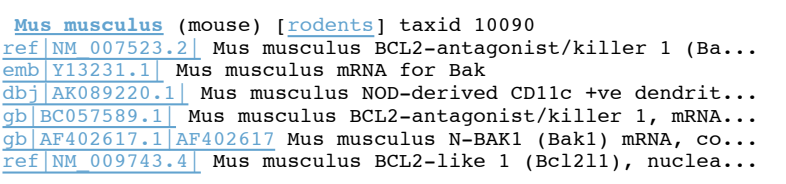
Mus musculus BCL2-like 1 (Bcl211), nuclea...

Rattus norvegicus (brown rat, ...) [rodents] taxid 10116 ref|NM 053812.1 Rattus norvegicus BCL2-antagonist/killer ... gb BC089784.1 Rattus norvegicus BCL2-antagonist/killer $1, \ldots$

Cricetulus griseus (Chinese hamsters) [rodents] taxid 10029 \begin{tabular}{c|l|l|l|l|l} 
gb & EF104644.1 & Cricetulus griseus Bak mRNA, complete cds \\
\cline { 1 - 2 } gb & EU596590.1 & Cricetulus griseus Bcl-xL mRNA, complete cds
\end{tabular} 
Saccoglossus kowalevskii [hemichordates] taxid 10224

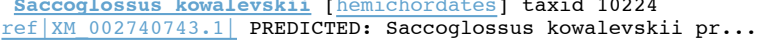

Saimiri boliviensis [primates] taxid 27679

gb|HM630305.1| Saimiri boliviensis BCL2-like protein 1 (BC...

Aotus vociferans (Spix's night monkey) [primates] taxid 57176

gb|HM630303.1| Aotus vociferans BCL2-like protein 1 (BCl2L...

Taeniopygia guttata (zebra finch) [birds] taxid 59729

ef|XM 002193086.1| PREDICTED: Taeniopygia guttata BCL2-li...

Felis catus (cat, ...) [carnivores] taxid 9685

Felis catus BCL2L1 (BCL2L1) mRNA, partial cds

Spermophilus tridecemlineatus [rodents] taxid 43179

gb|D0503423.1| Spermophilus tridecemlineatus Bcl-XL (Bcl-X...

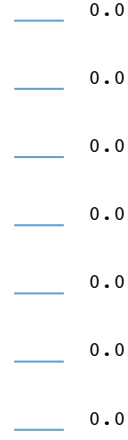

\section{Taxonomy Report}

root....

- Eumetazoa

. Cnidaria . Hexacorali

lla vectensis

Hydra magnipapillat

Hydra vulgaris

Deuterostomia

$\therefore$. Chordata $\ldots \ldots \ldots \ldots \ldots \ldots$

Tetrapoda

Amniota

Theria $\ldots \ldots \ldots \ldots \ldots \ldots$

Monodelp

. Laurasiatheria

. . . . Carnivora ... Caniformia

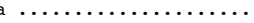

. . . . . . Ailuropoda melanoleuca ............

...... Canis lupus familiaris

. . . Cetartiodactyla

. . . . . . . Oovidae aries

..... . . Bos taurus

Equus caballus

. . . Euarchontoglires

$\therefore$. . . . Similatyrmes

. . . . . Callithrix jacchus

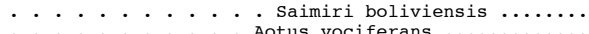

. . . . . Catarrhini

. . . . . Hominidae $\ldots \ldots \ldots \ldots \ldots \ldots$

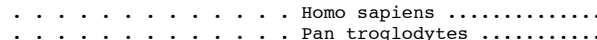

. Pan troglodytes

. Pongo abelii

Maca

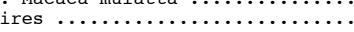

Oryctolagus cuniculus

Sciurognathi

- Murinae

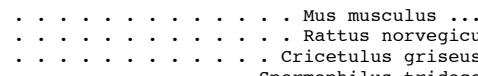

$\therefore$. . . . Rattus norvegicus

$\therefore \quad$ R Rattus norvegicus $\ldots \ldots \ldots$

$\therefore$. Spermophilus tridecemlineatus

$\therefore \therefore$. Neognathae .....

Gallus gallus .......

enopus

Xenopus (Silurana) tropicali

$\therefore$. Xenopus (Silurana)

$\therefore$ Eleuthenopus

.

$\therefore$. . Patiria miniata

. Saccoglossus kowalevski

synthetic construct.
106 hits 34 orgs

33 orgs [cellular organisms; Eukaryota; Fungi/Metazoa group

94 hits 32 orgs

$\begin{array}{ll}6 \text { hits } & 4 \text { orgs } \\ 2 \text { hits } & 2 \text { orgs [Anthozoa] }\end{array}$

1 hits 1 orgs [Scleractinia; Astrocoeniina; Acroporidae; Acropora]

1 hits 1 orgs [Actiniaria; Edwardsiidae; Nematostella]

4 hits 2 orgs [Hydrozoa; Hydroida; Anthomedusae; Hydridae]

2 hits 1 orgs

2 hits 1 orgs

88 hits 28 orgs [Bilateria; Coelomata]

84 hits 25 orgs 1 hits 1 orgs [Cephalochordata; Branchiostomidae; Branchiostoma]

83 hits 24 orgs [Craniata; Vertebrata; Gnathostomata; Teleostomi; Euteleostomi; Sarcop

80 hits 22 orgs

76 hits 20 orgs [Mammalia] Didelphimorphia; Didelphidae; Didelphinae; Monodelphis]

75 hits 19 orgs

19 hits 7 orgs

$\begin{aligned} 19 & \text { hits } \\ 5 \text { hits } & 3 \text { orgs }\end{aligned}$

4 hits 2 orgs

2 hits 1 orgs [Ursidae; Ailuropoda

2 hits 1 orgs [Canidae; Canis; Canis lupus]

1 hits 1 orgs [Feliformia; Felidae; Felinae; Felis]

12 hits 3 orgs

2 orgs [Ruminantia; Pecora]

1 hits 1 orgs [Caprinae; Ovis]

2 hits 1 orgs [Suina; Suidae; Sus]

2 hits 1 orgs [Perissodactyla; Equidae; Equus; Equus subg. Equus]

56 hits 12 orgs

43 hits 7 orgs [Primates; Haplorrhini]

6 hits 3 orgs

5 hits

4 hits

1 hits

37 hits

29 hits

29 hits

22 hits

4 hits
3 hits

3 hits

8 hits

13 hits

1 hits

12 hits

9 hits

hits

3 hits

2 hits

$\begin{array}{ll}2 & \text { hits } \\ 4 & \text { hits } \\ 3 & \text { hits }\end{array}$

4 hits

3 hits

1 hits

3 hits

2 hits

3 hits

2 hits

2
1 hits

1 hits

orgs [Callitrichinae; Callithrix]

1 orgs [Saimiriinae; Saimiri]

4 orgs

orgs [Hominoidea]

2 orgs

1 orgs [Homo]

orgs [Pan]

orgs [Ponginae; Pongo]

orgs [Cercopithecoidea; Cercopithecidae; Cercopithecinae; Macaca]

1 orgs [Lagomorpha; Leporidae; Oryctolagus]

4
3 orgs

orgs Muridae]

orgs [Mus; Mus]

orgs [Ratt

1 orgs [Cricetidae; Cricetinae; Cricetulus]

1 orgs [Sciuridae; Xerinae; Marmotini; Spermophilus]

2 orgs [Sauropsida; Sauria; Archosauria; Dinosauria; Saurischia; Theropoda; C

orgs [Galliformes; Phasianidae; Phasianinae; Gallus]

1 orgs [Passeriformes; Passeroidea; Estrildidae; Estrildinae; Taeniopygia]

orgs [Amphibia; Batrachia; Anura; Mesobatrachia; Pipoidea; Pipidae; Xenopod

1 orgs [Silurana]

2 orgs [Echinodermata

1 orgs [Echinozoa; Echinoidea; Euechinoidea; Echinacea; Echinoida; Strongyloc

1 orgs [Asterozoa; Asteroidea; Valvatacea; Valvatida; Asterinidae; Patirial

$\begin{array}{rr}1 & \text { hits } \\ 11 & \text { hits }\end{array}$

1 orgs [Placozoa; Trichoplax]

1 orgs [other sequences; artificial sequences] 


\section{Basic Local Alignment Search Tool}

Jump to Page Content

\section{Tax BLAST Report}

\section{Index}

- Lineage Repor

- Organism Repor

- Taxonomy Repor

- $\underline{\text { Help }}$

\section{Lineage Report}

root
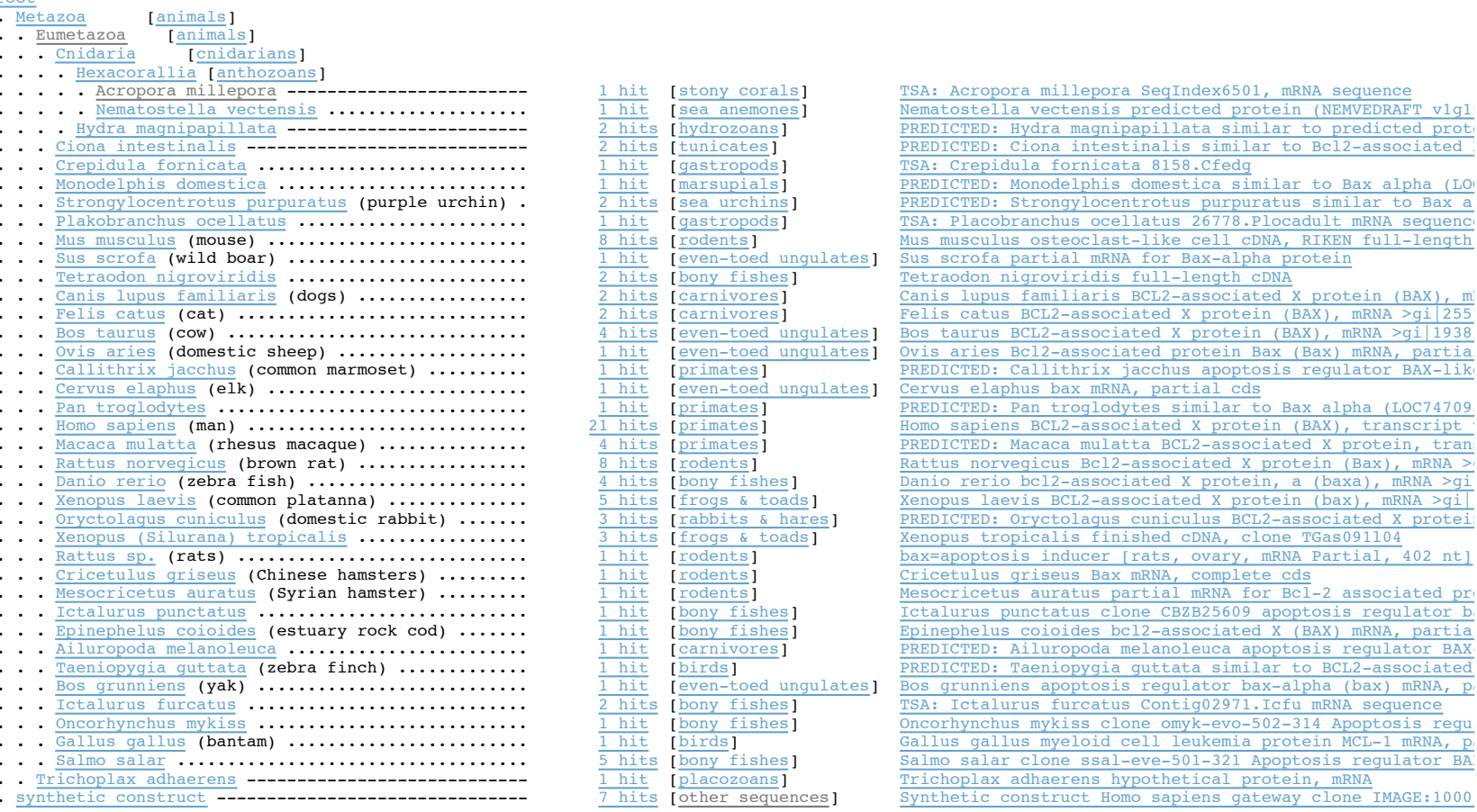

\section{Organism Report}

Acropora millepora [stony corals] taxid 45264

gb|EZ034459.1| TSA: Acropora millepora SeqIndex6501, mRNA ...

Nematostella vectensis [sea anemones] taxid 45351

ref|XM 001634806.1| Nematostella vectensis predicted prote...

Hydra magnipapillata [hydrozoans] taxid 6085

ref|XM 002157424.1 PREDICTED: Hydra magnipapillata simila...

ref XM 002158033.1 PREDICTED: Hydra magnipapillata simila...

Trichoplax adhaerens [placozoans] taxid 10228

ref|XM 002107647.1| Trichoplax adhaerens hypothetical prot...

Ciona intestinalis [tunicates] taxid 7719

ref|XM 002122967.1| PREDICTED: Ciona intestinalis similar ... dbj|AK116369.1| Ciona intestinalis cDNA, clone:citb036h13,...

Crepidula fornicata [gastropods] taxid 176853

Monodelphis domestica [marsupials] taxid 13616

ref|XM 001379887.1| PREDICTED: Monodelphis domestica simil... (...) [sea urchins] taxid 7668

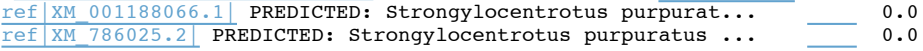

Plakobranchus ocellatus [gastropods] taxid 259542 gb|HP190622.1| TSA: Placobranchus ocellatus 26778.Plocadul...

Mus musculus (mouse) [rodents] taxid 10090

dbj|AK159233.1 Mus musculus osteoclast-like cell CDNA, RI... gb|AY095934.1| Mus musculus Bcl2-associated X protein kapp... ref NM 007527.3 Mus musculus BCL2-associated $\mathrm{x}$ protein (B... gb $\mid \mathrm{BC} 018228.1$ Mus musculus BCL2-associated X protein, mRN... gb abj AK013298.1 Mus musculus 10, 11 days embryo whole body... dbj AK149994.1 Mus musculus bone marrow macrophage CDNA, .. gb|L22472.1|MUSBAXA Mouse Bax alpha mRNA, complete cds 
Canis lupus familiaris (dogs) [carnivores] taxid 9615

Felis catus (cat, ...) [carnivores] taxid 9685

ef NM 001009282.1 Felis catus BCL2-associated x protein ... dbj $|\mathrm{AB} 080724.2|$ Felis catus mRNA for bax-protein, complete...

Bos taurus (cow, ...) [even-toed ungulates] taxid 9913

ref|NM 173894.1| Bos taurus BCL2-associated $x$ protein (BAX... gb 092569.1 BTU92569 Bos taurus apoptosis regulator bax-al... Sef XM 002701220.1 Bos taurus BCL2-associated X protein $(.$.

Ovis aries (domestic sheep, ...) [even-toed ungulates] taxid 9940 gb|AF163774.1 A|AF163774 Ovis aries BCl2-associated protein ...

Callithrix jacchus (common marmoset, ...) [primates] taxid 9483 ref|XM 002807856.1| PREDICTED: Callithrix jacchus apoptosi...

Cervus elaphus (elk) [even-toed ungulates] taxid 9860 GaF512030.1 Cervus elaphus bax mRNA, partial cds

Pan troglodytes [primates] taxid 9598

ref|XM 001155879.1 PREDICTED: Pan troglodytes similar to ...

Homo sapiens (man) [primates] taxid 9606

ref|NM 138761 3| Homo sapiens BCL2-associated $x$ protein (B... gb $\mathrm{BC} 014175,2$ Homo dbj|AK291076.1 Homo sapiens CDNA FLJ77115 complete cds, h. mb CR595189.1 full-length CDNA clone CS0DE013YL22 of Pla.. gb L22473.1 HUMBAXA Human Bax alpha mRNA, complete cds emb AJ417988.1 Homo sapiens mRNA for bax isoform psi (BAX... ref $\mid$ NM 138763.3 Homo sapiens BCL2-associated $x$ protein (B.. gb U19599.1 HSU19599 Human (BAX delta) mRNA, complete cds

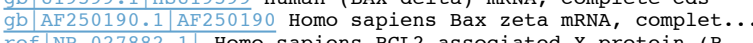
gb AF007826.1 AF007826 Homo sapiens bax epsilon mRNA, comp... ref NM 004324.3 Homo sapiens BCL2-associated $x$ protein $B$ gb L22474.1 HUMBAXB Human Bax beta mRNA, complete cds emb $\mid$ AJ586910.1 Homo sapiens partial mRNA for bax protein ... emb AJ586909.1 Homo sapiens partial mRNA for bax protein ... cef NM 138764.4 Homo sapiens BCL2-associated $\mathrm{X}$ protein (B... b AF247393.1 AF247393 Homo sapiens bax-sigma mRNA, comple... gb AY217036.1 Homo sapiens BCL2-associated X protein (BAX... ref $\mid$ NG 012191.1. Homo sapiens BCL2-associated x protein (B... gb $\mathrm{AC015871.7}$ Homo sapiens chromosome 15, clone RP11-81A1...

Macaca mulatta (rhesus macaque, ...) [primates] taxid 9544 ef XM 002801334.1 PREDICTED: Macaca mulatta BCL2-associa... ref XM 001112353.1 PREDICTED: Macaca mulatta BCL2-associa... ref XM 002801333.1 PREDICTED: Macaca mulatta BCL2-associa..

Rattus norvegicus (brown rat, ...) [rodents] taxid 10116 ref|NM 017059.1| Rattus norvegicus Bcl2-associated $\mathrm{x}$ prote... gb U49729.1 RRU49729 Rattus norvegicus rBax alpha mRNA, co... gb AF235993.1 AF235993 Rattus norvegicus Bax protein splic... \begin{tabular}{l|l|l|l} 
emb & FO229844.1 & Rattus norvegicus TL0ADA46YH24 mRNA sequence \\
\hline
\end{tabular} gb U59184.1 RNU59184 Rattus norvegicus Bax-alpha mRNA, par... gb U32098.1 RNU32098 Rattus norvegicus bax mRNA, partial cds ref XM 001060981.2 PREDICTED: Rattus norvegicus similar t... ref XM 001081479.2 PREDICTED: Rattus norvegicus similar t...

Danio rerio (zebra fish, ...) [bony fishes] taxid 7955 ref|NM 131562.2| Danio rerio bci2-associated x protein, a ... gb BC055592.1 Danio rerio bcl2-associated X protein, a, m... gb BC164990.1 Danio rerio bcl2-associated x protein, a, m... Xenopus laevis (common platanna, ...) [ [frogs \& toads] taxid 8355
ref|NM $001085635.1 \mid$ Xenopus laevis BCL2-associated X prote... gb AF288809.1 AF288809 Xenopus laevis bax mRNA, complete cds gb 1 BC169589.1 Xenopus laevis BCL2-associated X protein, m... $\begin{array}{lll}\text { gb BC169587.1 } & \text { Xenopus laevis BCL2-associated X prote } \\ \text { gb AY437085.1 } & \text { Xenopus laevis Bax mRNA, complete cds }\end{array}$

Oryctolagus cuniculus (domestic rabbit, ...) [rabbits \& hares] taxid 9986 ref|XM 002723696.1| PREDICTED: Oryctolagus cuniculus BCL2-...

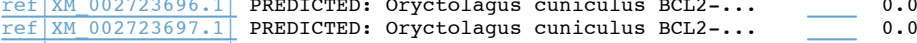

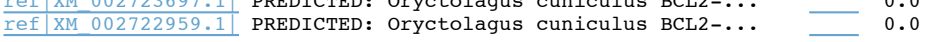

Xenopus (Silurana) tropicalis [frogs \& toads] taxid 8364 emb|CR855728.2| Xenopus tropicalis finished CDNA, clone TG... \begin{tabular}{l|l|l|l} 
ref & NM 203854.1 & Xenopus (Silurana) tropicalis BCL2-associ... \\
\hline gb & BC063201.1 Xenopus tropicalis BCL2-associated X protei...
\end{tabular}

Rattus sp. (rats) [rodents] taxid 10118 gb|S76511.1 bax=apoptosis inducer [rats, ovary, mRNA Part... Cricetulus griseus (Chinese hamsters) [rodents] taxid 10029 gb|EF104643.1| Cricetulus griseus Bax mRNA, complete cds Mesocricetus auratus (Syrian hamster, ...) [ [ rodents] taxid 10036
emb|AJ582075.1| Mesocricetus auratus partial mRNA for Bcl-... 

ynthetic construct N-EGFP/BCL2-associated ... Synthetic construct DNA, clone: pF1KB6979,... ynthetic construct Homo sapiens clone FLH1... Synthetic construct Homo sapiens clone FLH1... construct Homo sapiens clone FLHO...

pinephelus coioides (estuary rock cod, ... gb $\mid$ AY735005.1 | Epinephelus coioides bcl2-associated X (BAX...

Ailuropoda melanoleuca [Carnivores] taxid 9646

ref|XM 002917916.1| PREDICTED: Ailuropoda melanoleuca apop...

Taeniopygia guttata (zebra finch) [birds] taxid 59729

PREDICTED: Taeniopygia guttata similar...

Bos grunniens (yak) [even-toed ungulates] taxid 30521

gb|AY450350.1| Bos grunniens apoptosis regulator bax-alpha...

Ictalurus furcatus [bony fishes] taxid 66913

b|HP432380.1| TSA: Ictalurus furcatus Contig02971.Icfu mR...

gb GU588167.1 Ictalurus furcatus clone CBZF10821 apoptosi...

Oncorhynchus mykiss [bony fishes] taxid 8022

b BT074328.1| Oncorhynchus mykiss clone omyk-evo-502-314 ...

Gallus qallus (bantam, ...) [birds] taxid 9031

gb|AF120210.1|AF120210 Gallus gallus myeloid cell leukemia..

Salmo salar [bony fishes] taxid 8030

Salmo salar clone ssal-eve-501-321 Apoptosi...

Salmo salar clone ssal-rgf-501-217 Apoptosi...

Salmo salar clone ssal-evd-538-260 Apoptosi...

Salmo salar clone ssal-eve-507-043 Apoptosi...

Salmo salar clone ssal-eve-576-277 Apoptosi...

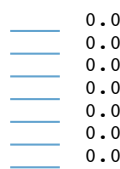

\section{Taxonomy Report}

root

Metazoa.

. Eumetazoa . Cnidaria

Hexacorallia

Acropora millepor

- Hydra magnipapillata

Coelomata

Deuterostomia

Chordata ..............

,

uteleostomi

Tetrapoda

.

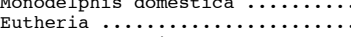

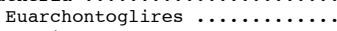

Glires

Muroidea.....

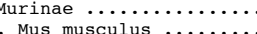

Rattus ................

Rattus norvegicus...

- Rattus sp.

Cricetulus griseus .

Oryctolagusetus auratus

Simiiformes

Callithrix jacchus .........

atarrhini..........

Pan troglodytes

Homo sapiens

.

Laurasiatheria

$$
\text { Bovidae }
$$$$
\text { . }
$$

.............

Ovis aries...

Caniformi

Canis lupus familiaris. . Ailuropoda melanoleuca.

Neognathae ........ Felis catus

.................

. Tallopygia guttata.

. Gapus gallus

Euteleostei

Tetraodon nigroviridi

- Epinephelus coioide

Oncorhynchus mykiss

. Salmo sala

Danio rerio.

- Ictalurus furcatus

Strongylocentrotus purpuratus

Apogastropoda
107 hits 39 orgs

100 hits 38 orgs [cellular organisms; Eukaryota; Fungi/Metazoa group]

99 hits 37 orgs
4 hits 3 orgs

4 hits

2 hit

1 hits

2 hits

95 hits

93 hits

91 hits

2 hits

89 hits

73 hits
65 hits
63 hits

1 hits

62 hit

49 hits

22 hits

19 hits
17 hits

8 hits

9 hits
8 hits
1 hits

1 hits

1 hit

1 hits

3 hits

27 hits

1 hits

26 hits

22 hits

$\begin{aligned} 1 & \text { hits } \\ 21 & \text { hits }\end{aligned}$

21 hits

13 hits

$\begin{aligned} & \\ 8 & \text { hits } \\ & \text { hits }\end{aligned}$

1 hits

7 hits

6 hits

5 hit

4 hits

1 hits

1 hits

1 hits

3 hits

2 hits

1 hits
2 hits

2 hits

1 hit

1 hits

8 hits

3 hits

16 hits

9 hits

3 hits

2 hits

1 hits

6 hits

1 hits

5 hits

7 hits

4 hits

1 hits

$\begin{array}{ll}1 & \text { hits } \\ 2 & \text { hits }\end{array}$

2 hits
2 hits
2 hits

orgs [Anthozoa]

orgs [Scleractinia; Astrocoeniina; Acroporidae; Acropora]

[Actiniaria; Edwardsiidae; Nematostella]

1 orgs [Hydrozoa;

32 orgs

1 orgs [Tunicata; Ascidiacea; Enterogona; Phlebobranchia; Cionidae; Ciona]

orgs [Craniata; Vertebrata; Gnathostomata; Teleostomi]

orgs [Sarcopterygii]

1 orgs

1 orgs [Metatheria; Didelphimorphia; Didelphidae; Didelphinae; Monodelphis]

8 orgs

18 orgs

6 orgs

5 orgs [Rodentia; Sciurognathi]

orgs [Muridae]

1 orgs [Mus; Mus]

2 orgs

1 orgs [unclassified Rattus]

1 orgs [Cricetidae]

orgs [Mesocricetus

1 orgs [Lagomorpha; Leporidae; Oryctolagus]

orgs [Primates; Haplorrhini]

1 orgs [Platyrrhini; Cebidae; Callitrichinae; Callithrix]

2 orgs [Hominoidea; Hominidae]

1 orgs [Pan]

1 orgs [Cercopithecoidea; Cercopithecidae; Cercopithecinae; Macaca]

8 orgs

1 orgs [Suina; Suidae; Sus]

4 orgs [Ruminantia]

3 orgs

orgs [Bovinae]

1 orgs

1 orgs [Caprinae; Ovis]

1 orgs [Cervidae; Cervinae; Cervus]

3 orgs

1 orgs [Canidae; Canis; Canis lupus]

1 orgs [Ursidae; Ailuropoda]

1 orgs [Feliformia; Felidae; Felinae; Felis]

2 orgs [Sauropsida; Sauria; Archosauria; Dinosauria; Saurischia; Theropoda; Coe

1 orgs [Passeriformes; Passeroidea; Estrildidae; Estrildinae; Taeniopygia]

orgs [Galliformes; Phasianidae; Phasianinae; Gallus]
orgs [Amphibia; Batrachia; Anura; Mesobatrachia; Pipoidea; Pipidae; Xenopodina

1 orgs [Xenopus]

7 orgs [Actinopterygii; Actinopteri; Neopterygii; Teleostei; Elopocephala]

4 orgs

2 orgs [Neognathi; Neoteleostei; Eurypterygii; Ctenosquamata; Acanthomorpha; Eua

1 orgs [Tetraodontiformes; Tetraodontoidei; Tetradontoidea; Tetraodontidae; Tetr

1 orgs [Perciformes; Percoidei; Serranidae; Epinephelinae; Epinephelus]

2 orgs [Protacanthopterygii; Salmoniformes; Salmonoidei; Salmonidae]

1 orgs [Oncorhynchus

1 orgs [Salmo]

orgs [Otocephala; Ostariophysi

1 orgs [Cypriniphysi; Cypriniformes; Cyprinoidea; Cyprinidae; Danio]

1 orgs

orgs

1 orgs [Echinodermata; Eleutherozoa; Echinozoa; Echinoidea; Euechinoidea; Echina

2 orgs [Protostomia; Mollusca; Gastropoda; Orthogastropoda] 
$\therefore$ Crepidula fornicata $\ldots \ldots \ldots \ldots \ldots \ldots \ldots \ldots, \quad 1 \mathrm{~h}$

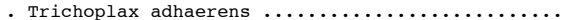
synthetic construct $\ldots \ldots \ldots \ldots \ldots \ldots \ldots \ldots \ldots, \quad 7$ hits
1 orgs [Caenogastropoda; Sorbeoconcha; Hypsogastropoda; Littorinimorpha; Calyptr. orgs [Heterobranchia; Euthyneura; Opisthobranchia; Sacoglossa; Placobranchoide 1 orgs [other sequences; artificial sequences] 